\title{
DISTRIBUCIÓN ESPACIAL DE BRACÓNIDOS (HYMENOPTERA) REPORTADOS EN EL ESTADO DE OAXACA
}

\author{
NANCY ALONSO-HERNÁNDEZ, ${ }^{1}$ JoSÉ ANTONIO SANCHEZ-GARCÍA, ${ }^{1 *}$ \\ José ISAAC FIGUEROA DE LA ROSA, ${ }^{2}$ VÍCTOR LÓPEZ-MARTÍNEZ, ${ }^{3}$ \\ LAURa MARTÍNEZ-MARTÍNEZ, ${ }^{1}$ RAFAel PÉREZ-PACHECO ${ }^{1}$ y CaRlos \\ GRANADOS-ECHEGOYEN ${ }^{1}$ \\ ${ }^{1}$ Instituto Politécnico Nacional, CIIDIR Unidad Oaxaca, Calle Hornos 1003, Santa Cruz, Xoxocotlán, \\ Oaxaca, México. C.P. 71230. \\ ${ }^{2}$ Instituto de Investigaciones Agropecuarias y Forestales. Universidad Michoacana de San Nicolás de \\ Hidalgo Km. 9.5 Carretera Morelia-Zinapécuaro, C.P. 58880. \\ ${ }^{3}$ Universidad Autónoma del Estado de Morelos, Facultad de Ciencias Agropecuarias. Av. Universidad \\ 1001, Col. Chamilpa Cuernavaca, Morelos, México. \\ *autor para correspondencia: <braconido2@yahoo.com>
}

Alonso-Hernández, N., Sanchez-García, J. A., Figueroa de la Rosa, J. I., López-Martínez, V., Martínez-Martínez, L., Pérez-Pacheco, R. y Granados-Echegoyen, C. 2014. Distribución espacial de bracónidos (Hymenoptera) reportados en el estado de Oaxaca. Acta Zoológica Mexicana (n.s.), 30(3): 564-594.

RESUMEN. Se presenta un listado de 300 morfoespecies, de las cuales se determinaron 83 especies y 146 géneros de Braconidae en el estado de Oaxaca. Se analizó la distribución espacial de los bracónidos registrados y se georreferenciaron los datos. Elaborándose un Sistema de Información Geográfica con los datos de cada especie registrada en literatura y material depositado en colecciones científicas. Las regiones con mayor abundancia de géneros fueron Costa, Valles Centrales e Istmo y las subfamilias más diversas fueron Agathidinae y Doryctinae. Para la selva mediana subperennifolia, se registró el mayor número de géneros (81), entre los más representativos se encontraron Heterospilus, Opius y Bracon. En zonas con temperaturas cálidas se registró el $85 \%$ de los géneros, incluyendo representantes de las subfamilias Alysiinae, Euphorinae, Doryctinae y Microgastrinae. El clima con mayor presencia de bracónidos fue el cálido subhúmedo y las subfamilias más abundantes fueron Doryctinae y Microgastrinae. En el clima semicálido subhúmedo se encontraron especialmente especímenes de Agathidinae. Los parasitoides se distribuyeron desde los 20 a 2900 msnm, pero el mayor número de registros se encontró a una altitud de $736 \mathrm{~m}$ y el género Heterospilus fue el mejor representado.

Palabras clave: parasitoides, georreferenciación, Sistemas de Información Geográfica, Braconidae.

Alonso-Hernández, N., Sanchez-García, J. A., Figueroa de la Rosa, J. I., López-Martínez, V., Martínez-Martínez, L., Pérez-Pacheco, R. \& Granados-Echegoyen, C. 2014. Spatial distribution

Recibido: 12/12/2013; aceptado: 11/06/2014. 
of braconids (Hymenoptera) records from the state of Oaxaca. Acta Zoológica Mexicana (n.s.), 30(3): 564-594.

ABSTRACT. A list of 300 morphospecies of Braconidae from State of Oaxaca is presented, of these 83 species and 146 genera were identified. In the study the spatial distribution of braconid was analyzed, records and data were georeferenced. A Geographic Information System with the species recorded from literature and material deposited in scientific collections was developed. The regions with the highest abundance of genera were Costa, Central Valleys and Istmo, the subfamilies most diverse were represented by Agathidinae and Doryctinae. The vegetation with highest incidence of records was the evergreen tropical forest that included 81 genera, the most representative genera were Heterospilus, Opius, and Bracon. The distribution of Braconidae in warm temperatures represented $85 \%$ of the genera, being the subfamilies Alysiinae, Euphorinae, Doryctinae, and Microgastrinae the most representative. The climate with major presence of braconids was the warm humid and the most abundant subfamilies were Doryctinae and Microgastrinae. In the semi-warm humid climate there were found especially specimens of the subfamily Agathidinae. The parasitoids were distributed from 20 to $2900 \mathrm{~m}$ of altitude, but the greatest number of records were found at the height of $736 \mathrm{~m}$ and the genus Heterospilus was the best represented.

Key words: parasitoids, georeference, Geographic Information System, Braconidae.

\section{INTRODUCCIÓN}

El desarrollo de los Sistemas de Información Geográfica (SIG), ha impulsado el análisis de la distribución espacial aplicada a la ecología de los insectos (Moral-García et al. 2004). Esto con el fin de conocer cómo interactúan las especies con su ambiente y proporcionar un medio simple y flexible del modelado de las relaciones existentes (Briggs 2005, Jerrett et al. 2005). Esta información puede proporcionar conocimientos sobre competencia intraespecífica, atracción mutua y dispersión de poblaciones que permiten caracterizar y analizar la distribución de insectos (Ellsbury et al. 1998, Park \& Obrycki 2004). Los atributos ecológicos y geográficos en la distribución espacial de los insectos son parámetros requeridos para desarrollar planes de muestreo en campo y ningún muestreo es viable si no se entiende antes la distribución espacial. Los SIG mediante el uso de programas de cómputo permiten analizar datos georreferenciados, tales como la densidad de una especie, tipo de vegetación, tipo de clima y de suelo, mismos que al ser incorporados en el sistema proporcionan mapas superpuestos que permiten tener una mejor representación de los individuos en estudio (Barrera 2008). Actualmente, los programas de manejo integrado de plagas requieren del uso de programas computacionales especializados para conocer la distribución de insectos benéficos y perjudiciales (Vílchez 2000). En el estado de Oaxaca, las avispas parasitoides de la familia Braconidae resaltan su importancia debido a que se les ha encontrado parasitando a diversas especies plagas (Sánchez et al. 2010a).

Actualmente existen varios trabajos en donde se cita la diversidad de Braconidae para el estado de Oaxaca; por ejemplo, Sánchez et al. (2003a) reportaron una revisión de los géneros de esta familia para el estado y registraron 21 subfamilias, 75 géneros y 26 especies. Posteriormente Sánchez et al. (2005) determinaron nueve especies 
de bracónidos asociados a plagas y realizaron un estudio faunístico de éstos en la región de la Sierra Sur y Costa del estado. En tanto que Mercado (1996) realizó un estudio sobre los himenópteros parasitoides asociados a Dendroctonus frontalis Zimn en Tlaxiaco. Asimismo, Ojeda (2005) realizó una investigación sobre los bracónidos asociados al cultivo de alfalfa en Santa Cruz Xoxocotlán y registró 20 géneros de 11 subfamilias. Sánchez et al. (2009) registró un aumento de la diversidad, citando 127 géneros pertenecientes a 24 subfamilias; este incremento fue debido principalmente por la aportación de los trabajos de Morales \& Sánchez (2007) quienes realizaron un estudio en la localidad de Pluma Hidalgo. Por otro lado Cruz (2009) y Martínez et al. (2008) estudiaron a los himenópteros parasitoides de Santa María Yavesía, Sierra Norte de Oaxaca; asimismo Sánchez et al. (2010a) reportan 15 especies de parasitoides asociados a plagas en el estado. Recientemente Coronado-Blanco y ZaldívarRiverón (2014) registraron 704 especies en 318 géneros en México y para Oaxaca 136 géneros y 61 especies. Otra parte importante del conocimiento de la diversidad de este grupo de insectos ha sido a través de las revisiones de colecciones nacionales y extranjeras (González et al. 2003, Labougle 1980, Quicke \& Kruft 1995, Sánchez et al. 1998, 2003a, 2003b, 2005, 2008, 2009, 2010a, 2010b, Sánchez \& López 2000, Wharton \& Mercado 2000); sin embargo, aun con el esfuerzo realizado hasta el momento, los trabajos taxonómicos referentes a la familia Braconidae para Oaxaca son insuficientes, debido a la enorme biodiversidad del estado, en gran parte dado por la gran variedad climática y geográfica.

El comportamiento y distribución de insectos benéficos y perjudiciales es afectado directamente por factores ambientales bióticos y abióticos. El cambio climático afecta la distribución, el ciclo de vida, la composición de las comunidades y la función del ecosistema de los insectos (Easterling et al. 2000, Bale et al. 2002). Los cambios de temperatura pueden influenciar la biología de las especies componentes de un ecosistema, provocando la desestabilización en la dinámica poblacional que puede conducir a la extinción de una parte del mismo. Uno de los principales efectos del calentamiento global serán los cambios acelerados en la distribución de especies. Muchos insectos tienen rangos geográficos que no se limitan directamente por la vegetación, pero si por la temperatura. Existe evidencia de que el cambio climático puede influir en la distribución de los insectos fitófagos y particularmente en lepidópteros (Batalden et al. 2007, Cannon 1998, Kiritani 2006, Lastuvka 2009, Parmesan et al. 1999). El cambio climático o calentamiento global podría adelantar las épocas del año y aumentar la posibilidad de migración de insectos (Lastuvka 2009, Woiwod 1997, Zhou et al. 1995) y ocasionar cambios de adaptabilidad de los mismos, por lo que se hace necesario conocer la distribución espacial actual de los insectos. Ante esta situación el presente trabajo contempló la creación de un Sistema de Información Geográfica que incluyó datos de distribución, tanto de las especies registradas en la literatura como de aquellos especímenes alojados en colecciones científicas de ins- 
tituciones de investigación; con esta información se analizó la distribución espacial de las especies sobre capas geográficas de regiones, distritos, vegetación, climas, temperaturas, relieve y carreteras del estado de Oaxaca. Esta información permitirá detectar regiones y distritos poco o nada explorados, los cuales podrán proporcionar información para realizar futuros estudios de distribución.

\section{MATERIAL Y MÉTODOS}

Oaxaca es una entidad estatal que posee una superficie de $95,364 \mathrm{~km}^{2}$ y se ubica al sur de la República Mexicana. Se encuentra dividido en ocho regiones socioeconómicas: Cañada, Costa, Istmo, Mixteca, Papaloapan, Sierra Norte, Sierra Sur y Valles Centrales; su territorio comprende un alta diversidad de suelos, clima y vegetación (García-Mendoza et al. 2004). La geomorfología característica en cada una de sus regiones determina la existencia de una gran heterogeneidad de hábitats (Jerez 2000).

Revisión de bibliografía y colecciones científicas. Se recaudó información contenida en las etiquetas de los ejemplares de la familia Braconidae depositados en la colección entomológica del Centro Interdisciplinario de Investigación para el Desarrollo Integral Regional (CIIDIR) - Oaxaca del Instituto Politécnico Nacional. Adicionalmente se revisaron las colecciones del Colegio de Posgraduados - Campus Montecillo, Universidad Autónoma de Chapingo (UACH), Instituto Tecnológico del Valle de Oaxaca (ITVO) y del Instituto Nacional de Investigaciones Forestales, Agrícolas y Pecuarias (INIFAP) de Oaxaca. De los especímenes revisados, se obtuvieron los datos de colecta, tomando en cuenta la información de localidad y coordenadas geográficas. Se realizó una revisión de literatura especializada producida entre los años 1937 y 2013, se tomaron referencias que incluyeran información de presencia de bracónidos para el estado, así como datos útiles para georreferenciación. Otras fuentes de información incluidas fueron el Catalogo de Bracónidos de Oaxaca elaborado por Sánchez-García et al. (2009) y datos recopilados y no publicados por el Dr. José Antonio Sánchez García del CIIDIR, Oaxaca.

Análisis y sistematización de la información. Se elaboró una base de datos en Microsoft Office Excel 2010 donde se integró la información de los ejemplares de bracónidos revisados en las colecciones entomológicas y de la revisión de literatura científica. Se incluyeron para cada registro de distribución los campos de nombre científico, localidad, municipio, latitud, longitud y elevación. Adicionalmente, con la información obtenida se actualizó la base de datos de bracónidos elaborada por el Dr. José Antonio Sánchez García del CIIDIR, Oaxaca, donde se corrigió y agregó con mayor precisión las colectas reportadas con anterioridad. Para ello se utilizó la cartografía de Oaxaca, el software gratuito Google Earth $\odot$ y el Anuario estadístico del Instituto Nacional de Estadística y Geografía (INEGI). Con lo anterior se generó una base de datos final en el programa Paradox versión 11.0 que incluyó los siguien- 
tes campos: orden, familia, subfamilia, tribu, género, especie, subespecie, autor, año, país, estado y/o provincia, región, localidad, fecha de colecta, número de individuos, tipo de vegetación, colector, planta hospedera, familia hospedera, nombre común, organismo huésped/presa, familia huésped/presa, determinador, colección, año de catalogación, cita bibliográfica, latitud, longitud y altitud; mismos que fueron útiles para la representación gráfica de cada uno de los ejemplares registrados.

Georreferenciación de datos y análisis espacial. De acuerdo a la metodología de Santiaguillo-Hernández et al. (2010) se utilizó el sistema de información geográfica con coordenadas latitud y longitud en grados sexagesimales para ubicar los registros de distribución en las capas geográficas de regiones, distritos, vegetación, climas, temperatura, relieve y carreteras del estado, en las que se sobrepusieron los registros de distribución de Braconidae.

Inicialmente se añadieron las temáticas cartográficas del estado de Oaxaca al programa ArcGIS 10.1 en forma de capas digitalizadas, se definió el sistema de coordenadas geográficas WGS_1984 (Meridiano de Greenwich) con la herramienta de gestión de datos en ArcTool Box para que cada capa tuviera una proyección.

Las tablas de atributos geográficos que contenían las coordenadas geográficas fueron importadas desde Microsoft Excel 2013 al programa ArcGIS ArcMap ${ }^{\mathrm{TM}} 10.1$ (ESRI® 2012). Se definieron las coordenadas para cada campo geográfico para generar una tabla de atributos desde ArcGIS, éstas fueron exportadas a formato dBase para que el programa asignará un número de identificación a cada dato. Para conocer la relación existente de cada registro con los parámetros ecológicos y geográficos de las subfamilias se procedió a realizar la unión de datos de cada capa basados en la localización espacial, con lo que se generó una tabla de atributos de coincidencia de datos con cada capa cartográfica.

Finalmente se elaboraron mapas digitalizados sobre cada temática empleada y cuadros de distribución geográfica en los que se describe el número de géneros y especies representadas para realizar el análisis espacial.

\section{RESULTADOS}

Se logró reunir información de 300 morfoespecies de la familia Braconidae, de ese material se determinaron 24 subfamilias, 146 géneros y 83 especies; esperando que en el futuro se pueda identificar la totalidad del material. Se obtuvieron 222 registros correspondientes a 2245 ejemplares determinados, según la clasificación de $\mathrm{Yu}$ et al. (2012), seguida por Coronado-Blanco (2013) (Cuadro 1). La cifra de géneros se incrementó en $6.45 \%$ de los 136 reportados por Coronado-Blanco \& ZaldívarRiverón (2014) a esta nueva cifra de 146. La cifra de especies también se incrementó en $26.50 \%$, de 61 que menciona Coronado-Blanco y Zaldívar-Riverón (2014) a esta nueva cifra de 83. Las subfamilias de Braconidae mejor representadas fueron Doryctinae y Microgastrinae con 22 y 18 géneros respectivamente. En cuanto a especies 


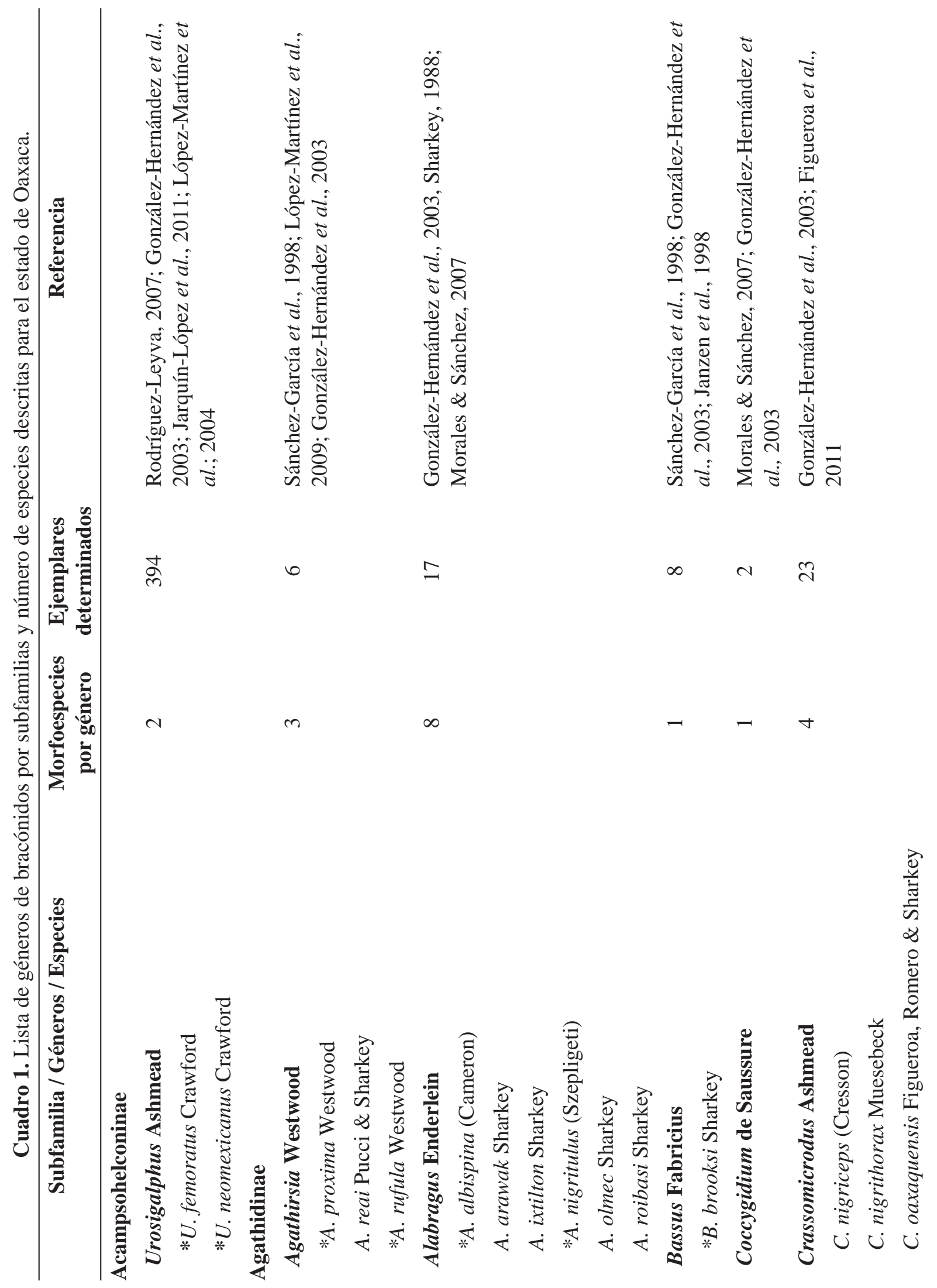




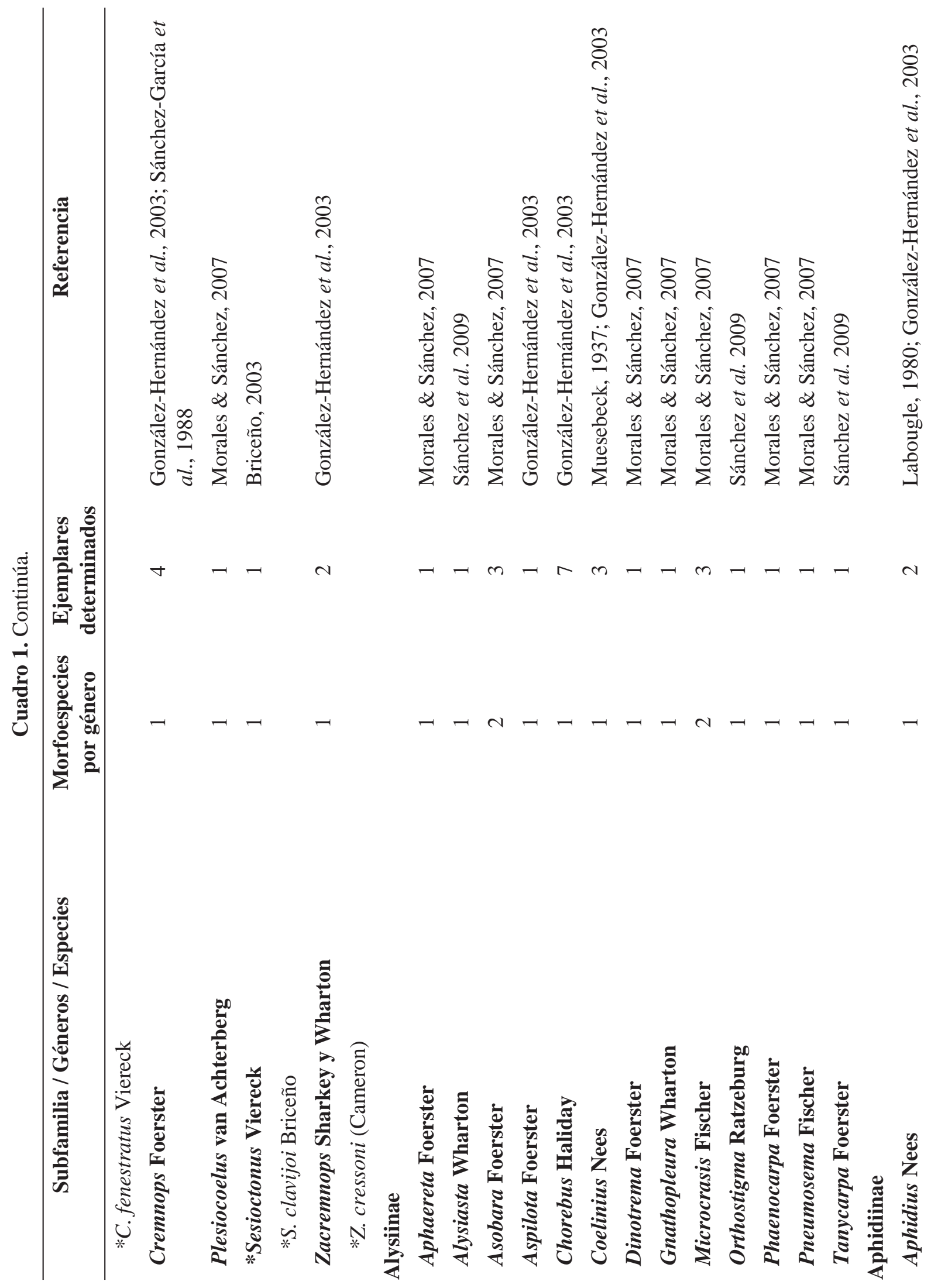


Acta Zool. Mex. (n.s.) 30(3) (2014)

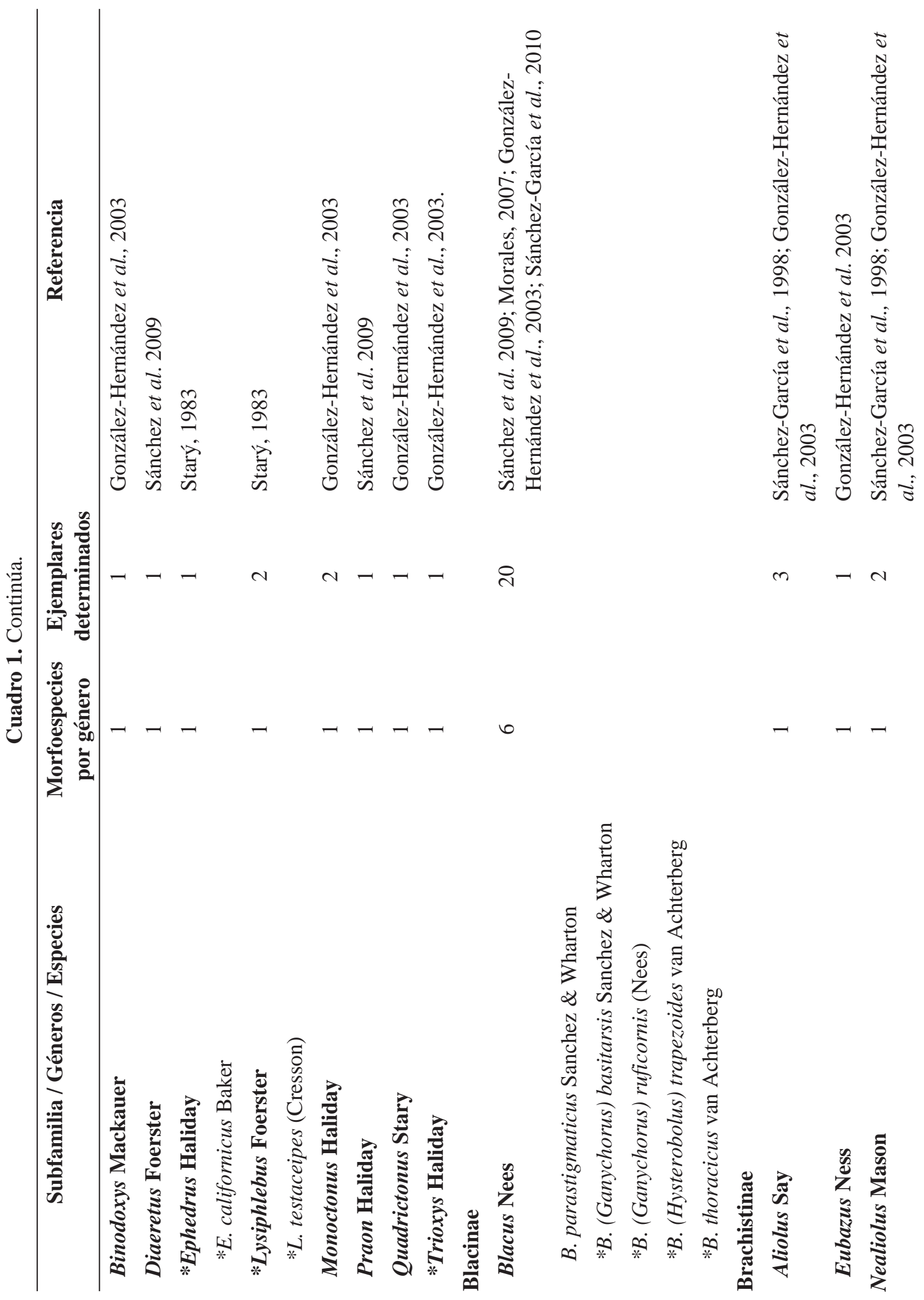




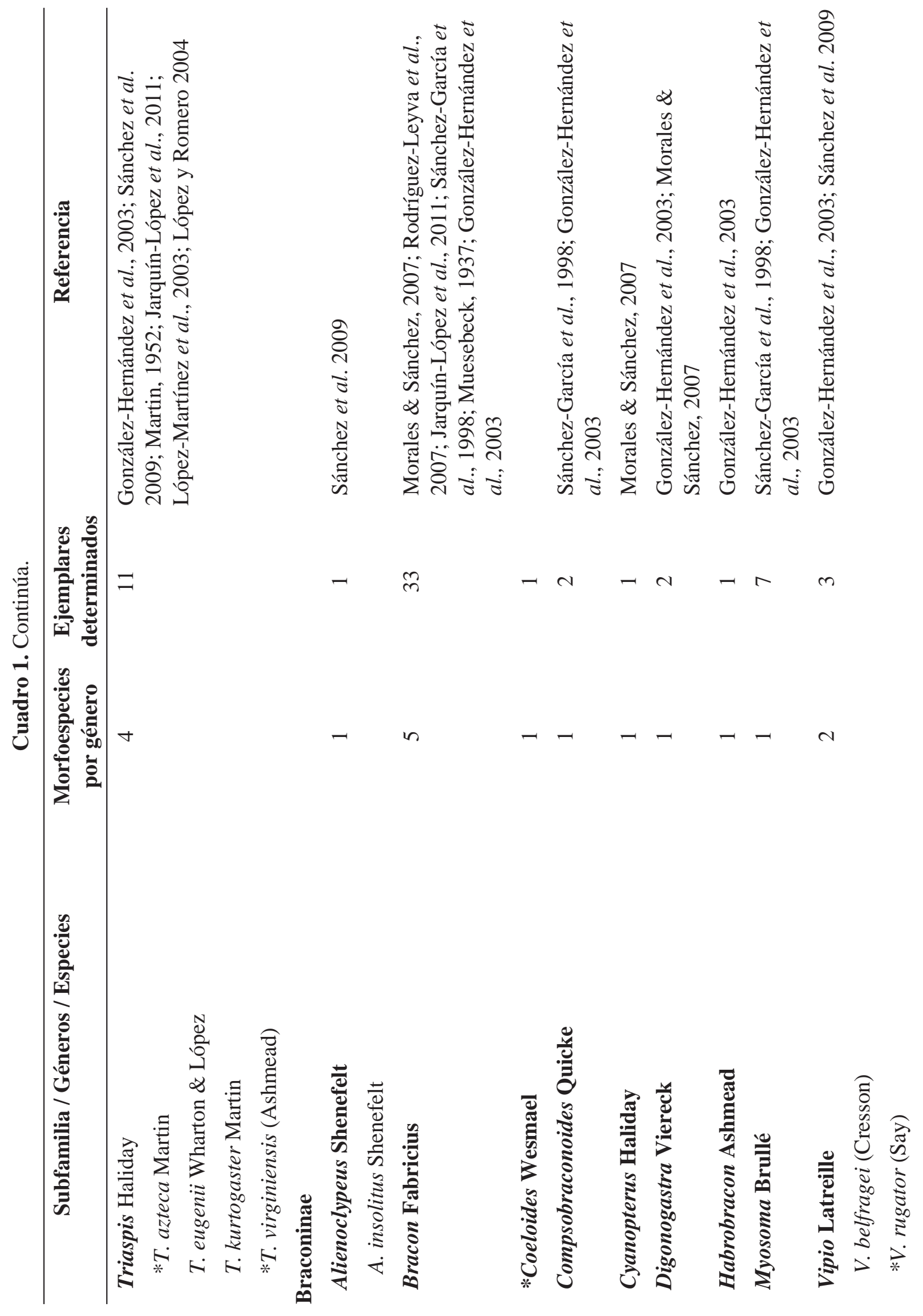


Acta Zool. Mex. (n.s.) 30(3) (2014)

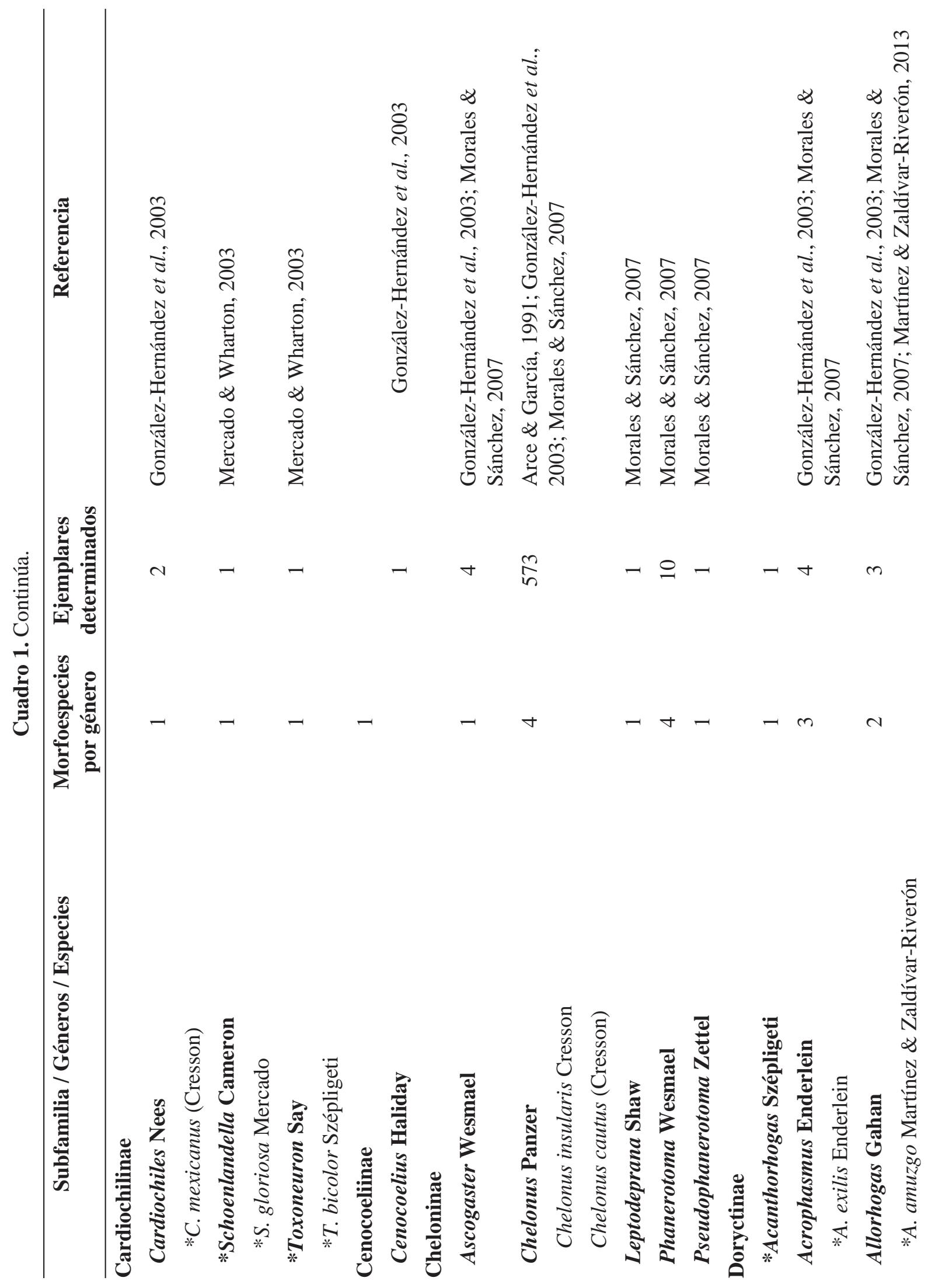




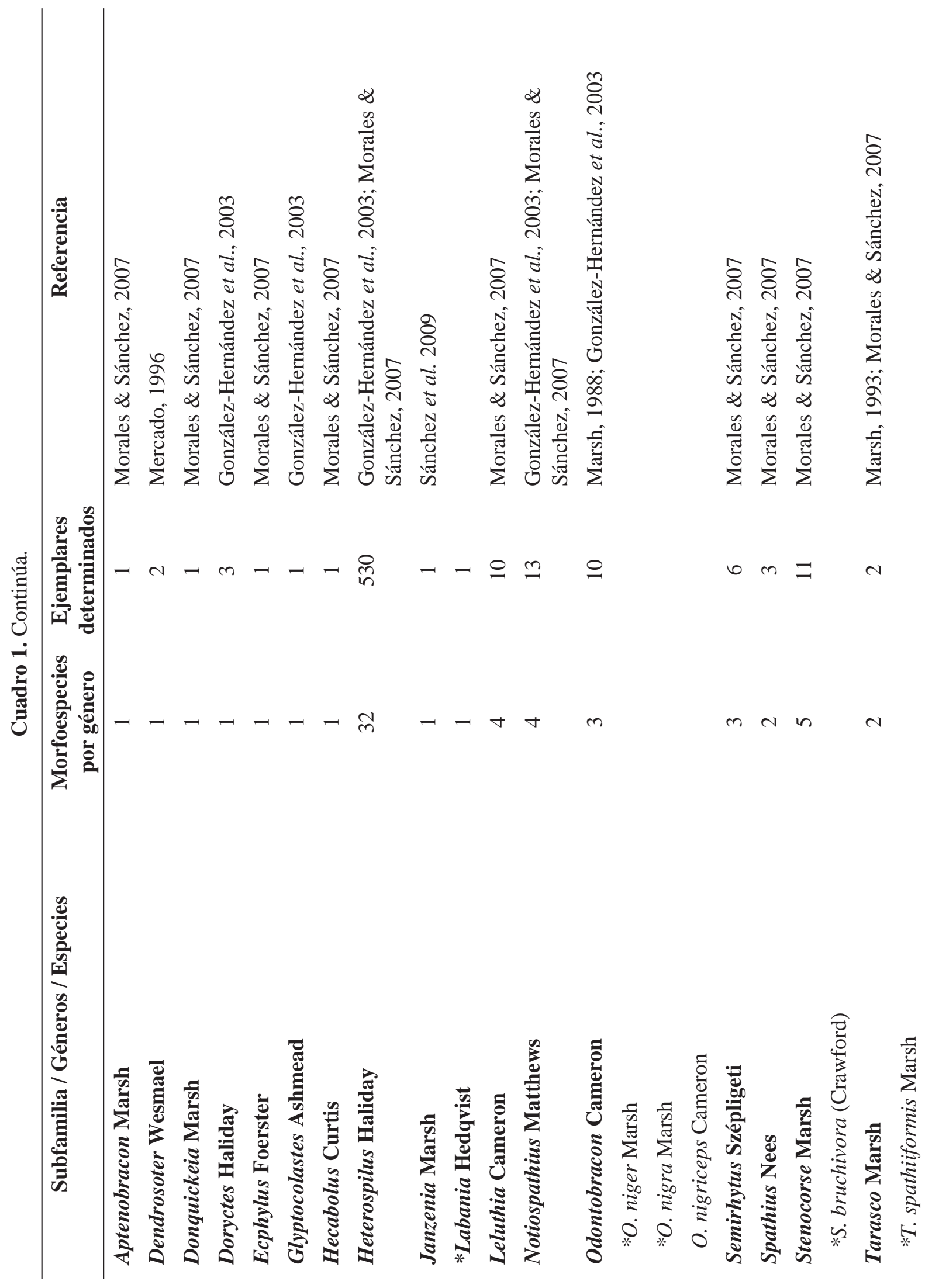




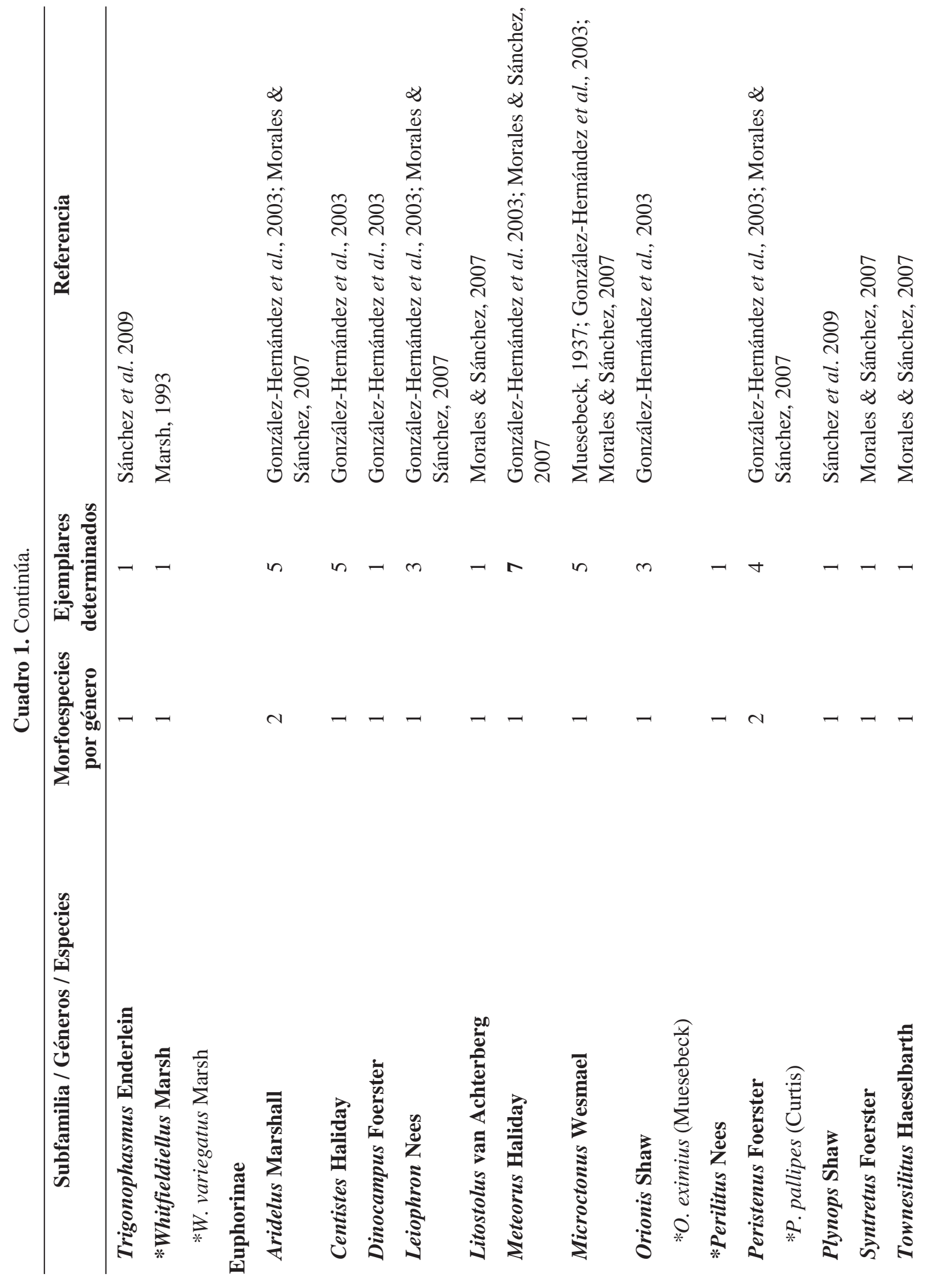




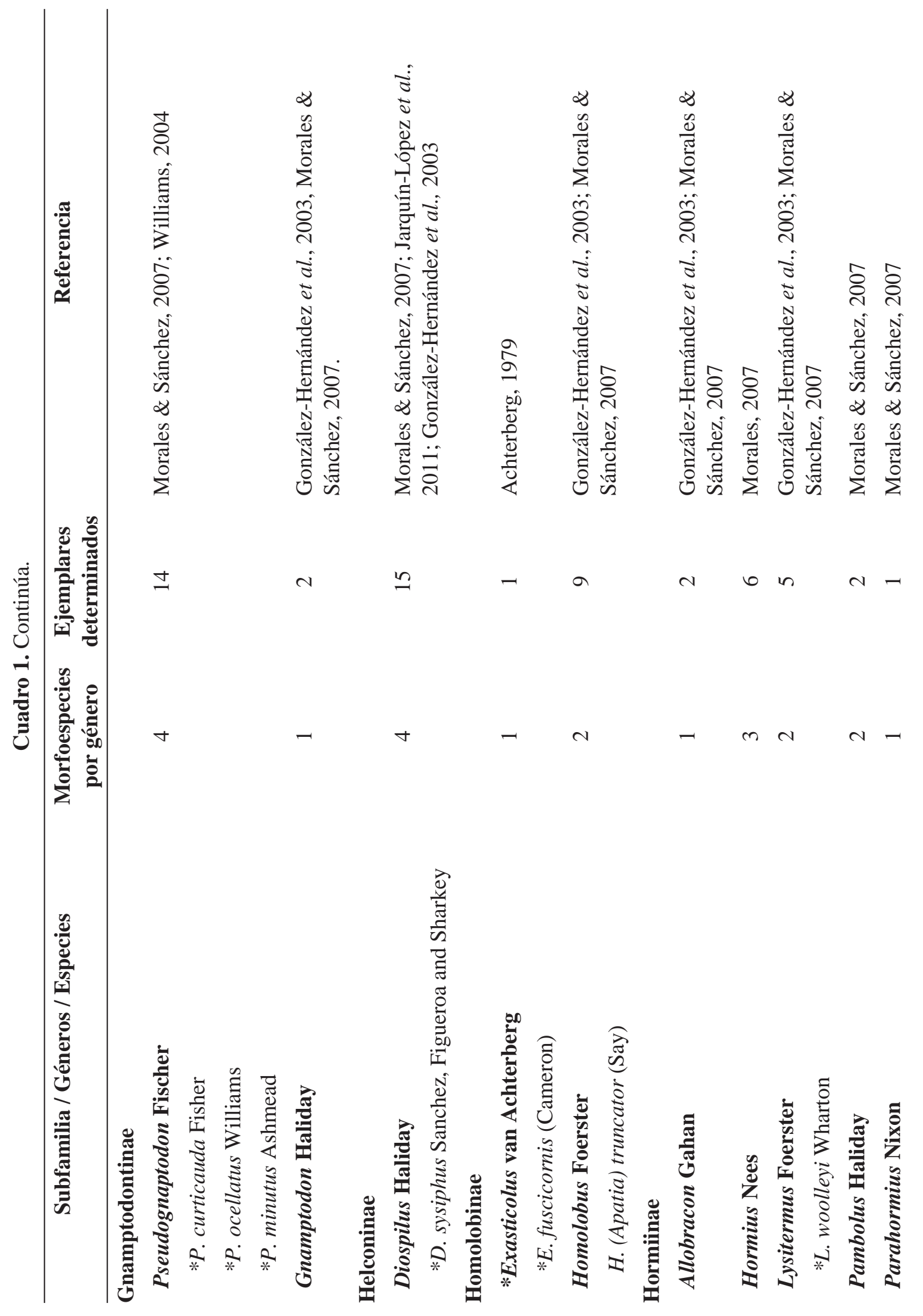




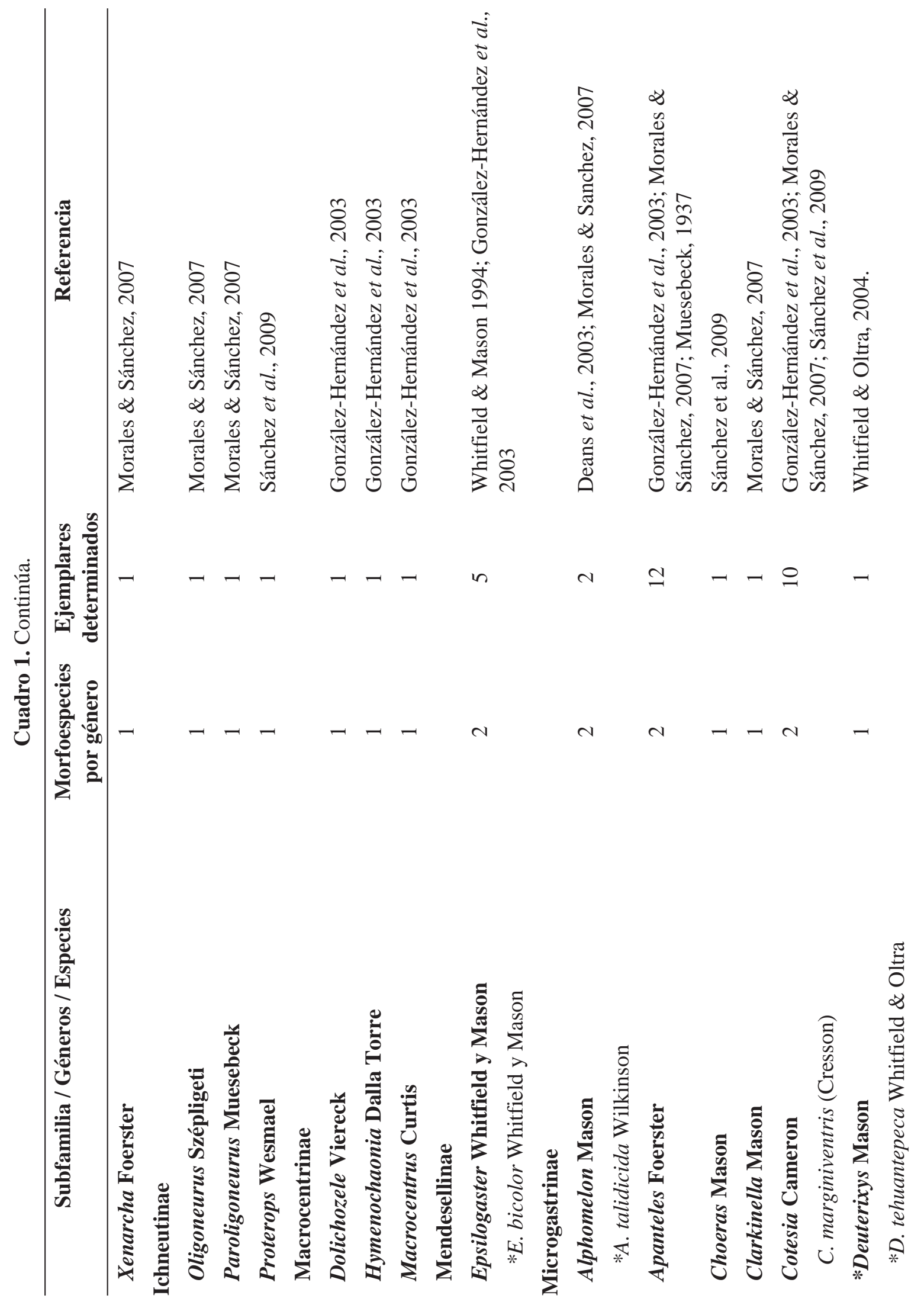




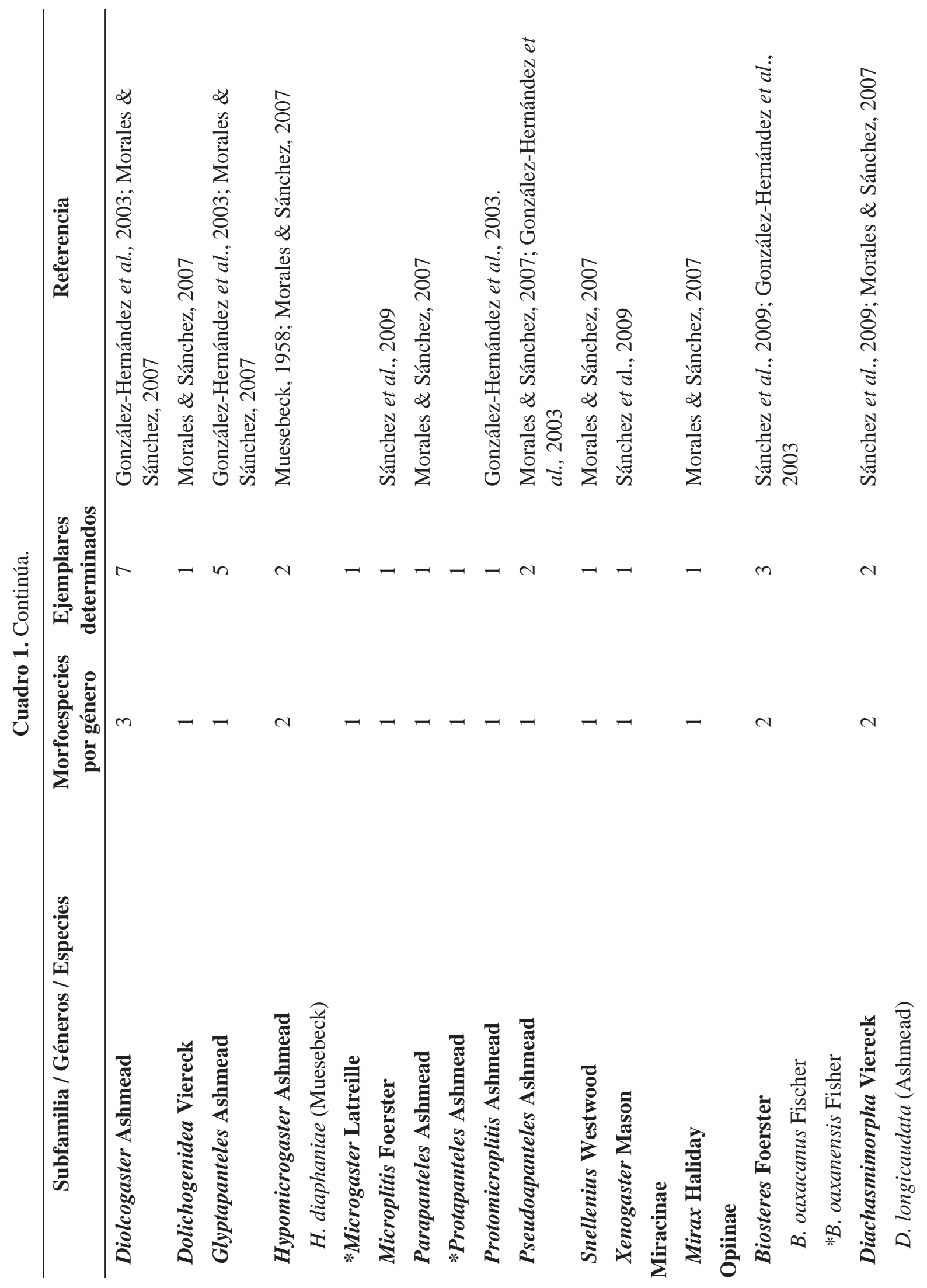




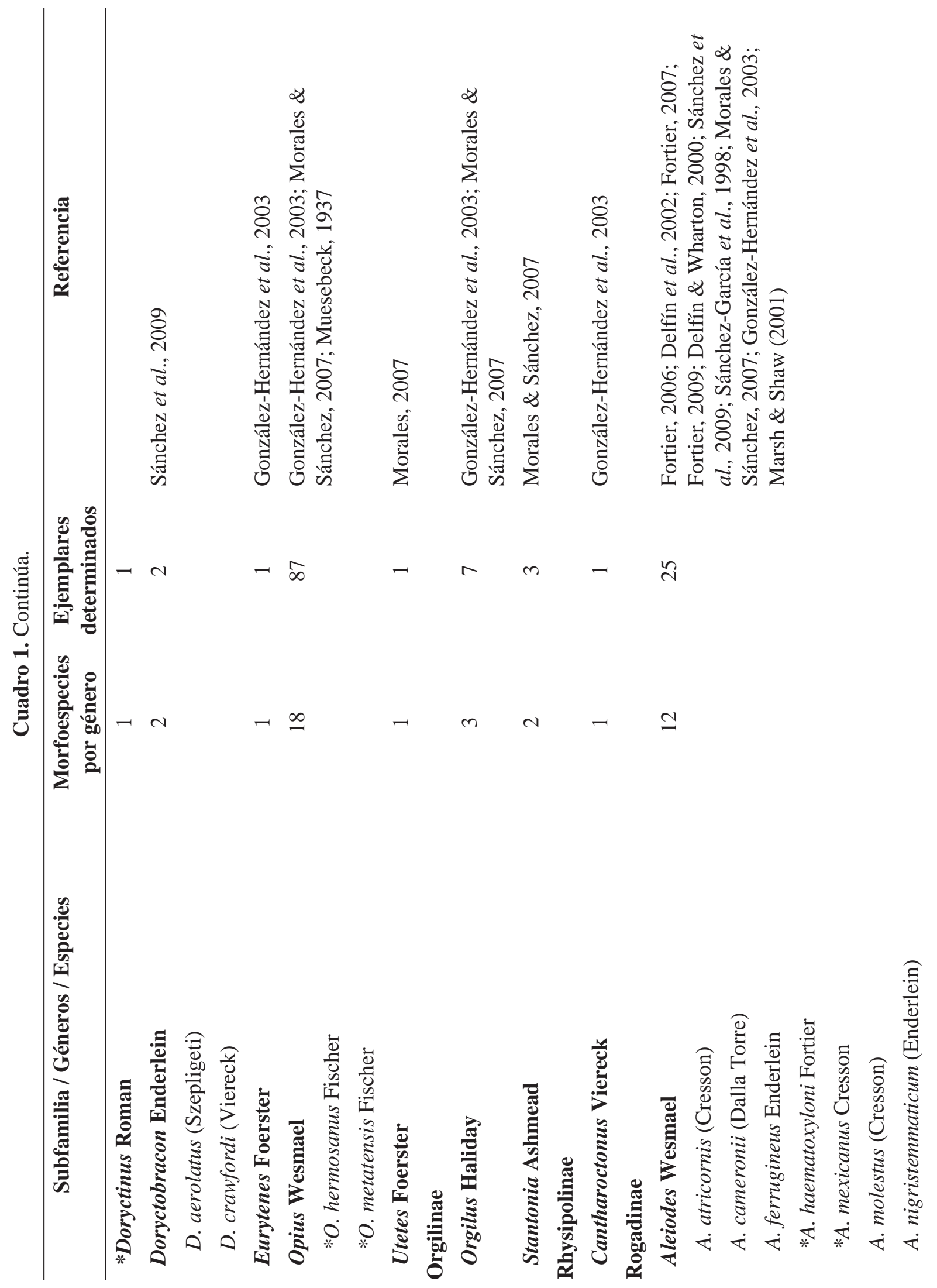




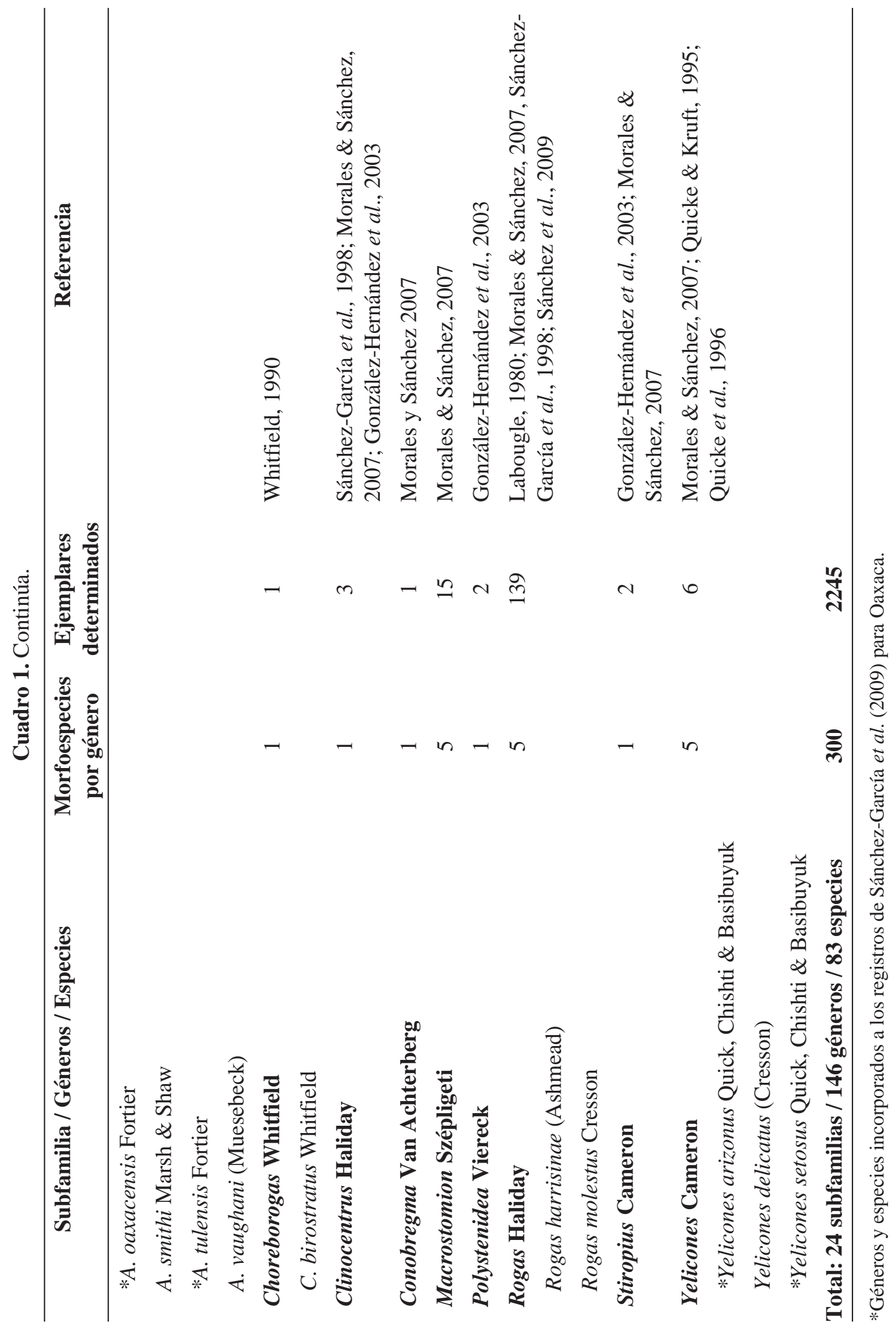


determinadas, las subfamilias Rogadinae, Agathidinae y Helconinae fueron las que tuvieron un mayor número con 17, 16 y 11 especies, respectivamente.

A continuación se indican los resultados de los análisis espaciales de Braconidae por cada capa analizada (regiones, distritos y carreteras, vegetación, climas, temperatura, relieve) para el estado de Oaxaca.

Análisis espacial por regiones. Las regiones con mayor incidencia de registros fueron la Costa, Valles Centrales y el Istmo de Oaxaca. La mayor representatividad de géneros en cuanto a número de registros de distribución de Braconidae para la región Costa fue el género Heterospilus (Doryctinae), seguido de Diolcogaster (Microgastrinae), Macrostomion (Rogadinae), Phaenocarpa (Alysiinae), Notiospathius, Stenocorse, Leluthia (Doryctinae) y Diospilus (Helconinae). Para la región Valles Centrales fueron los géneros Chelonus (Cheloninae), Urosigalphus (Acampsohelconinae), Rogas y Aleiodes (Rogadinae). En el Istmo de Oaxaca se registraron a Crassomicrodus (Agathidinae) y Odontobracon (Doryctinae) como las más representativas (Fig. 1).

Análisis espacial por distritos y carreteras. El distrito del estado de Oaxaca que presentó la mayor representatividad de bracónidos fue Pochutla con 78 géneros lo

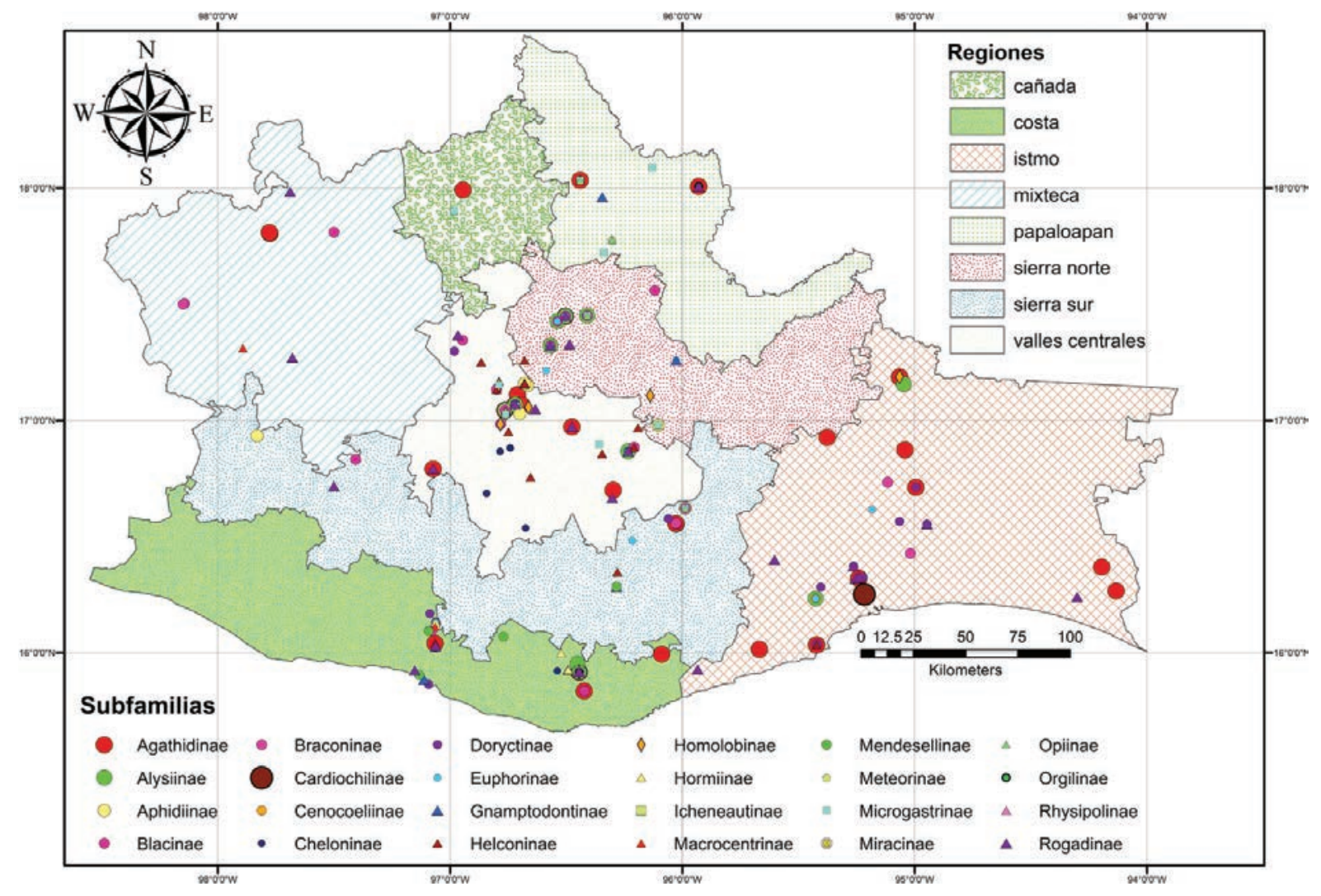

Figura 1. Registros de distribución de bracónidos sobre regiones del estado de Oaxaca. 
que representó un $53.42 \%$ del total de géneros reportados; el distrito Centro de la región valles Centrales registró 24 géneros; el distrito de Ixtlán, Tuxtepec y Tehuantepec pertenecientes a las regiones Sierra Norte, Papaloapan e Istmo estuvieron representados por aproximadamente 20 géneros cada uno. Mientras que los distritos de Choapam, Nochixtlán, Teotitlán, Teposcolula y Jamiltepec no presentaron reportes de distribución de especímenes de la familia Braconidae. La mayor concentración de registros de distribución se dispone en las cercanías de las carreteras que unen a la ciudad de Oaxaca con las de Cuicatlán, Puerto Escondido, Puerto Ángel, Totontepec y Tuxtepec; así como con los grandes sistemas montañosos Sierra Norte, Sur y Transístmica (Fig. 2).

Análisis espacial por vegetación. Las áreas de vegetación que presentaron mayor incidencia de registros fueron selva mediana subperennifolia (SMSP) con 81 géneros, agricultura de temporal con 29 y bosque de Pino con 26. En SMSP se localizan los géneros Heterospilus, Leluthia, Notiospathius y Stenocorse (Doryctinae), Opius (Opiinae), Bracon (Braconinae), Macrostomion (Rogadinae) y Phanerotoma (Cheloninae). En agricultura de riego se encontró a Bracon (Braconinae) y Urosigalphus (Acampsohelconinae). En agricultura de temporal los géneros Aleiodes y Rogas (Ro-

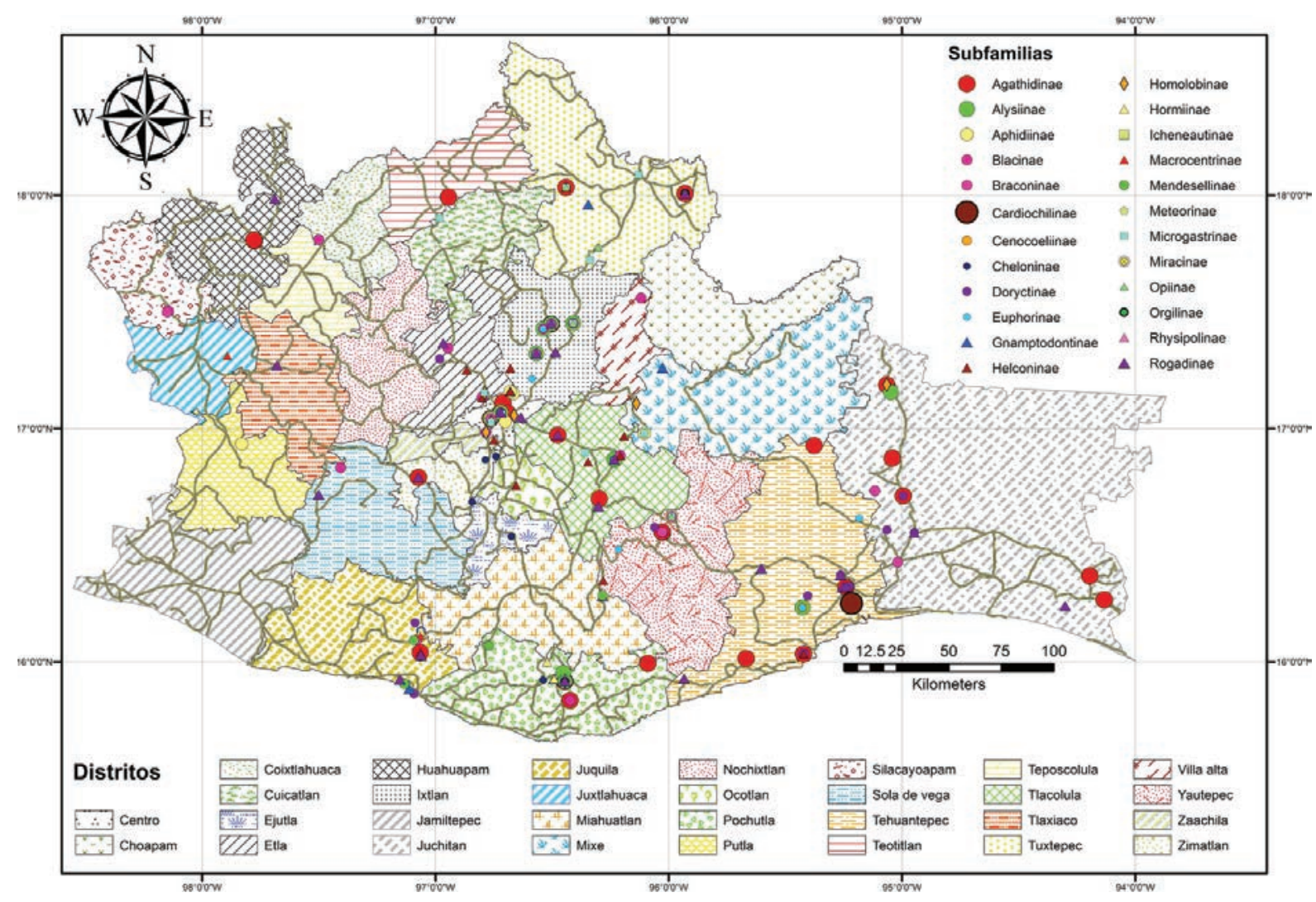

Figura 2. Registros de distribución de bracónidos sobre distritos y carreteras del estado de Oaxaca. 
gadinae) y Bracon (Braconinae). En bosque de pino, pastizal cultivado y selva baja caducifolia están representados por Urosigalphus (Acampsohelconinae), Alabagrus y Crassomicrodus (Agathidinae) (Fig. 3).

Análisis espacial por climas. El clima que presentó mayor presencia de parasitoides fue el cálido subhúmedo con 99 géneros. Chelonus y Heterospilus fueron los que reportaron mayor número de ejemplares determinados con 558 y 530, respectivamente. También en este clima se registraron los géneros Opius, Bracon, Macrostomion, Crassomicrodus, Notiospathius, Phaenocarpa, Leluthia, Stenocorse, Rogas, Diospilus y Odontobracon. El clima húmedo presentó dos géneros Alabagrus y Opius con ocho registros cada uno. Para el área semicálida subhúmeda se reportaron los géneros $\mathrm{Bra}$ con y Urosigalphus. El clima semiárido templado, semiárido cálido y templado subhúmedo coinciden con la presencia de los géneros Rogas, Aleiodes y Blacus (Fig. 4). Análisis espacial por temperaturas. Los bracónidos registrados en el estado de Oaxaca tienen mayor afinidad a temperaturas cálidas, en este clima se encontraron 106 géneros, en los más importantes se incluyen: Heterospilus, Bracon, Crassomicrodus, Alabagrus, Aleiodes, Stenocorse, Apanteles, Rogas, Diospilus, Phaenocarpa, Leluthia, Macrostomion, Orgilus, Myosoma, Notiospathius, Odontobracon y Opius.

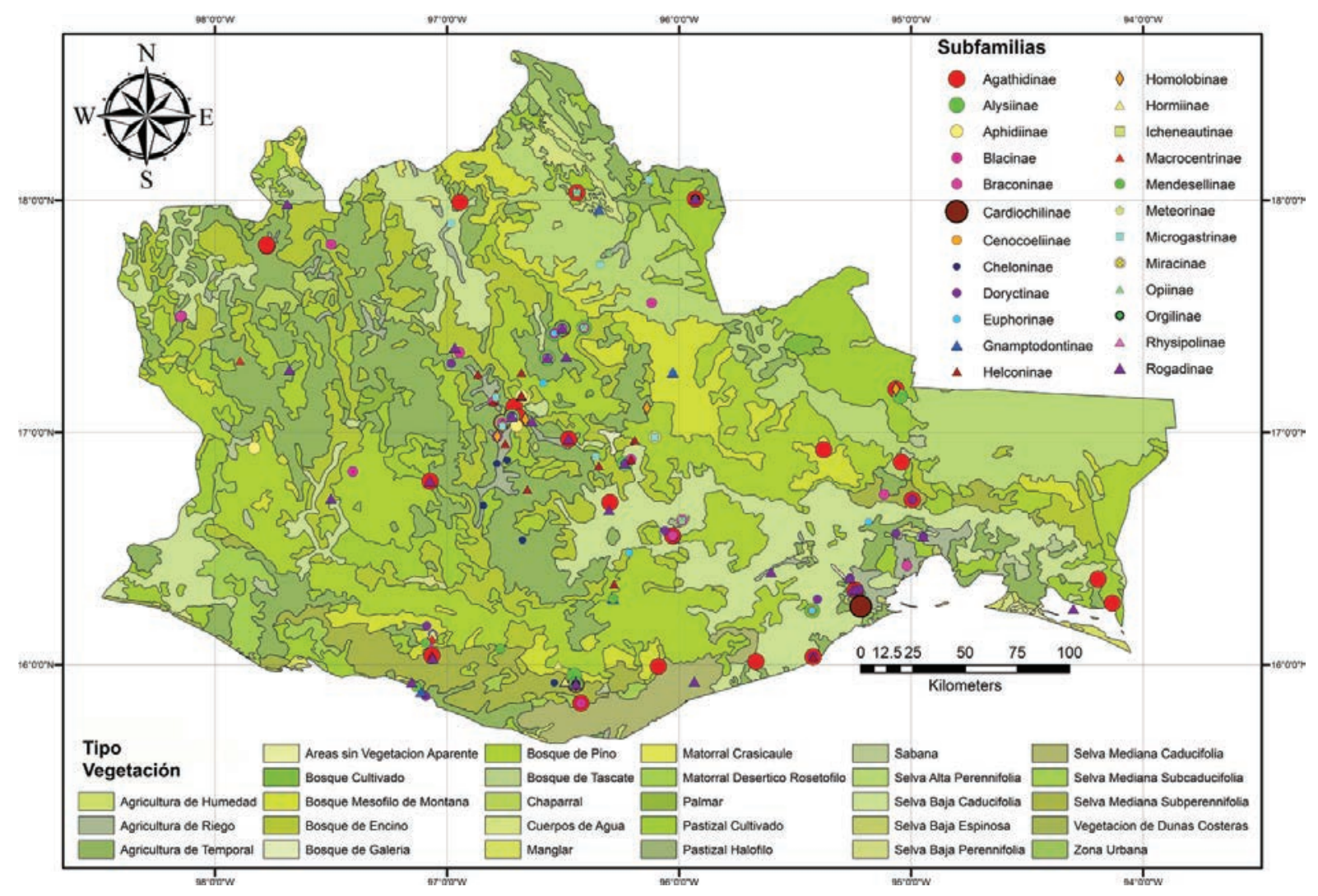

Figura 3. Registros de distribución de bracónidos sobre vegetación del estado de Oaxaca. 


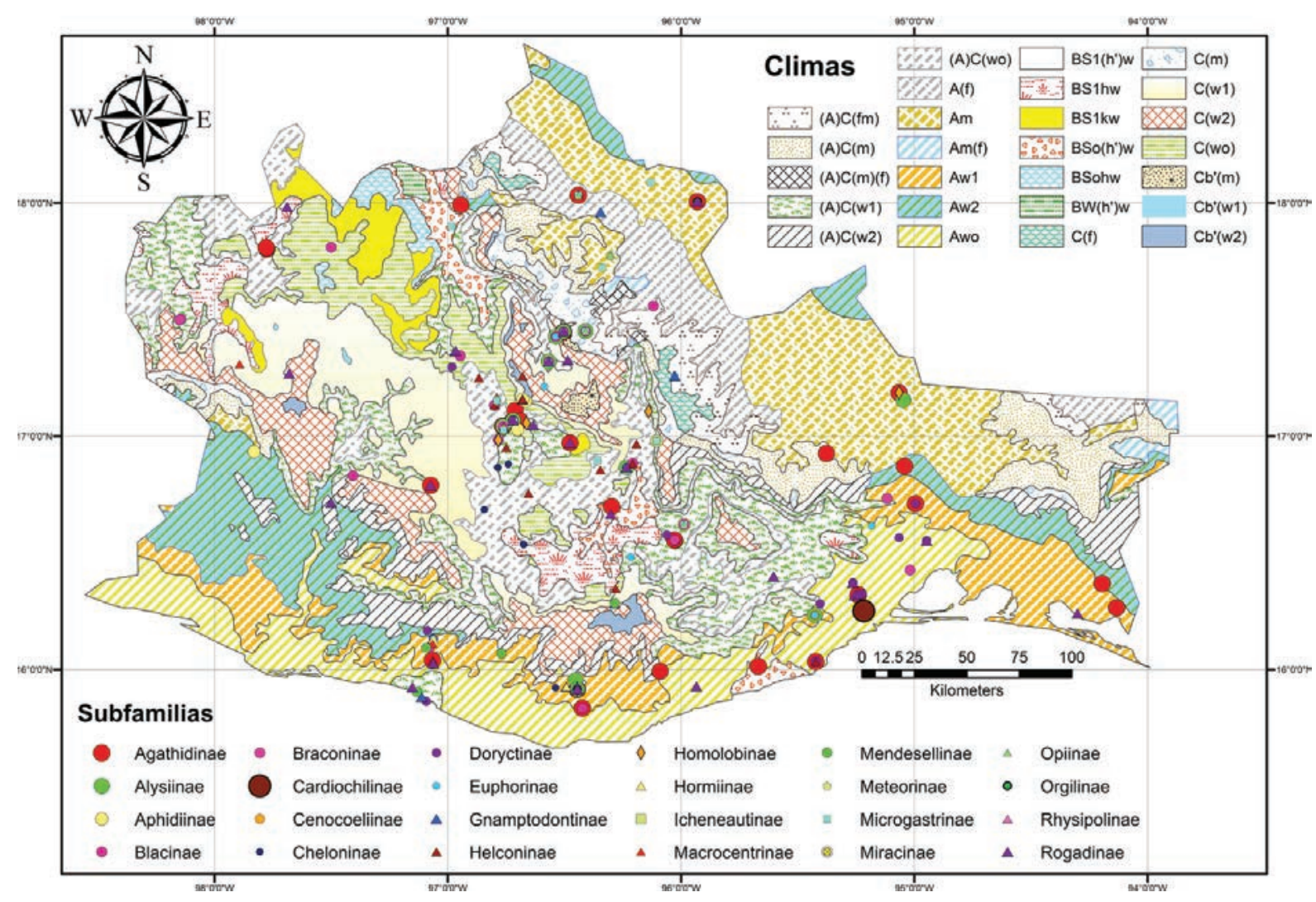

Figura 4. Registros de distribución de bracónidos sobre climas del estado de Oaxaca.

Los géneros Aleiodes, Bracon y Urosigalphus tienen concurrencia en el clima semicálido. En el caso del clima templado se registraron a Blacus y Rogas (Fig. 5).

Análisis espacial por relieve. Se encontró que los bracónidos se distribuyeron desde los 20 a 2,900 m, pero el mayor número de registros se encontró en el género Heterospilus en altitudes de $736 \mathrm{~m}$; entre los 1500-1600 m los géneros con mayor representatividad fueron Chelonus, Homolobus, Lysitermus y Cotesia. Entre los 20-60 y 55-200 m se encontró al género Opius y a Aleiodes, y Crassomicrodus respectivamente (Fig. 6). Para el rango de 1400-1600 m se registró a Aleiodes y Urosigalphus. En alturas de 1600-1800 m se detectó a Crassomicrodus. Entre 2200-2400 m el género representativo fue Alabagrus; en tanto que para el rango de 2600-2800 se encontraron a Blacus, Centistes y Chorebus. En el estado de Oaxaca se han registrado altitudes superiores a los $3720 \mathrm{~m}$; sin embargo, no se tienen registros de que a estas altitudes este grupo de insectos pueda sobrevivir.

\section{DISCUSIÓN}

Delfín-González \& León-Burgos (1997) mencionan que Yucatán es un estado con una diversidad de bracónidos similar a la de Oaxaca e indican la existencia 21 subfa- 


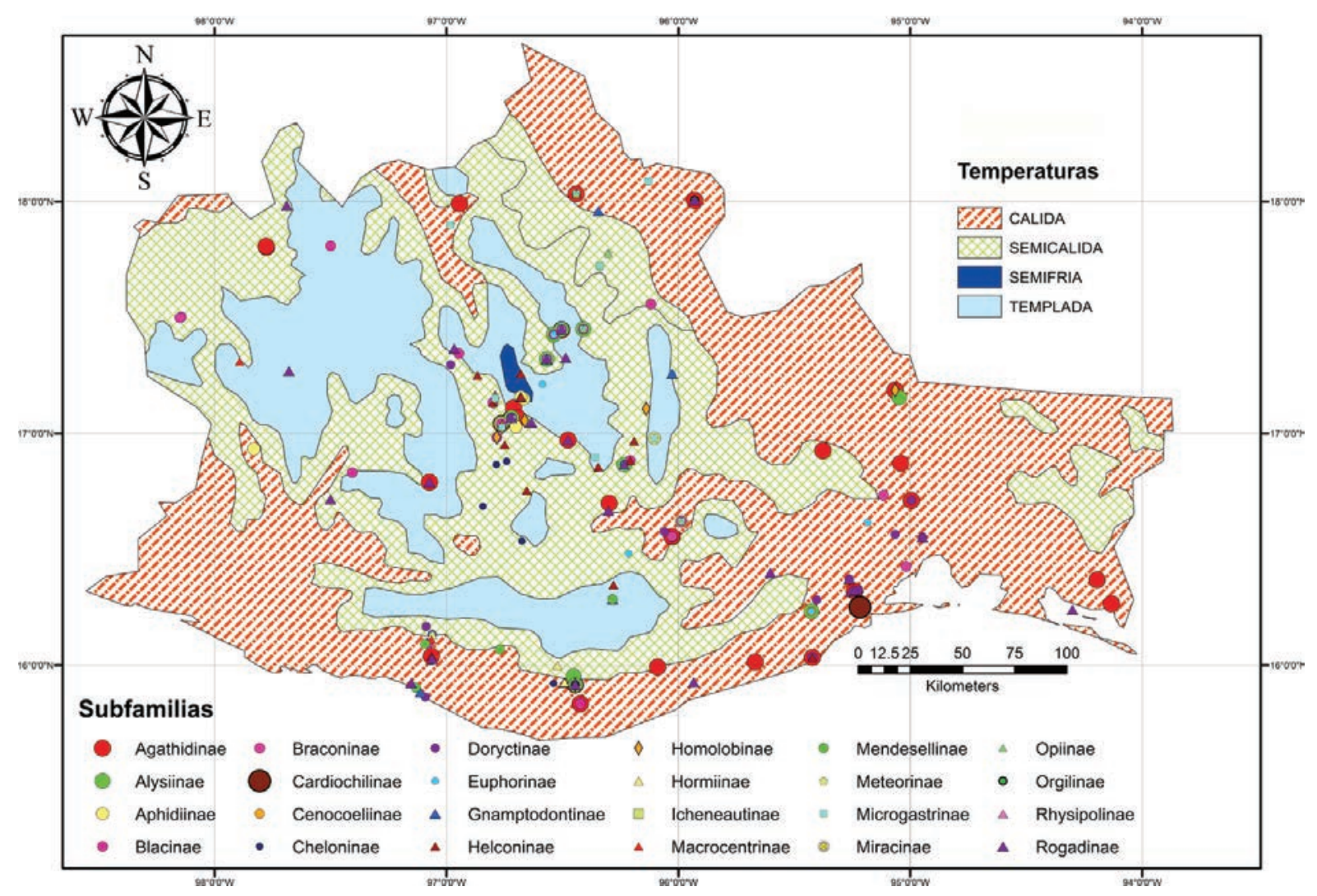

Figura 5. Registros de distribución de bracónidos sobre temperaturas del estado de Oaxaca.

milias y 78 géneros, lo que demuestra la diversidad de esta familia en zonas tropicales. Los géneros más diversos y abundantes mencionados en ese estudio incluyeron Apanteles, Bracon, Cardiochiles, Chelonus, Digonogastra, Heterospilus, Opius y Phanerotoma, géneros muy comunes y con alta representatividad en el estado oaxaqueño; asimismo reportan a Neoneurinae, subfamilia que hasta el momento no se reporta para Oaxaca. Por otro lado Delfín-González et al. (2002) registran por primera vez a la subfamilia Sigalphinae en México y reportan 27 nuevos registros de subfamilias para todo el país, de las cuales 25 subfamilias son registros para Yucatán, con lo que incrementó el conocimiento de bracónidos para México a 277 géneros y 190 para el estado de Yucatán. Por otra parte comentan que el $68.4 \%$ de los géneros examinados fueron representados en una sola comunidad vegetal. En el presente trabajo se reporta a la subfamilia Rhysipolinae que no ha sido registrada para Yucatán. Cauich-Kumul et al. (2012) mencionan la existencia de 83 especies en 29 géneros en Yucatán de las cuales 62 especies son nuevos registros para todo el país. Por otra parte Coronado-Blanco \& Zaldívar-Riverón (2013) mencionan que los estados con mayor número de géneros registrados son Yucatán con 194, Tamaulipas con 149 y Oaxaca con 136. 


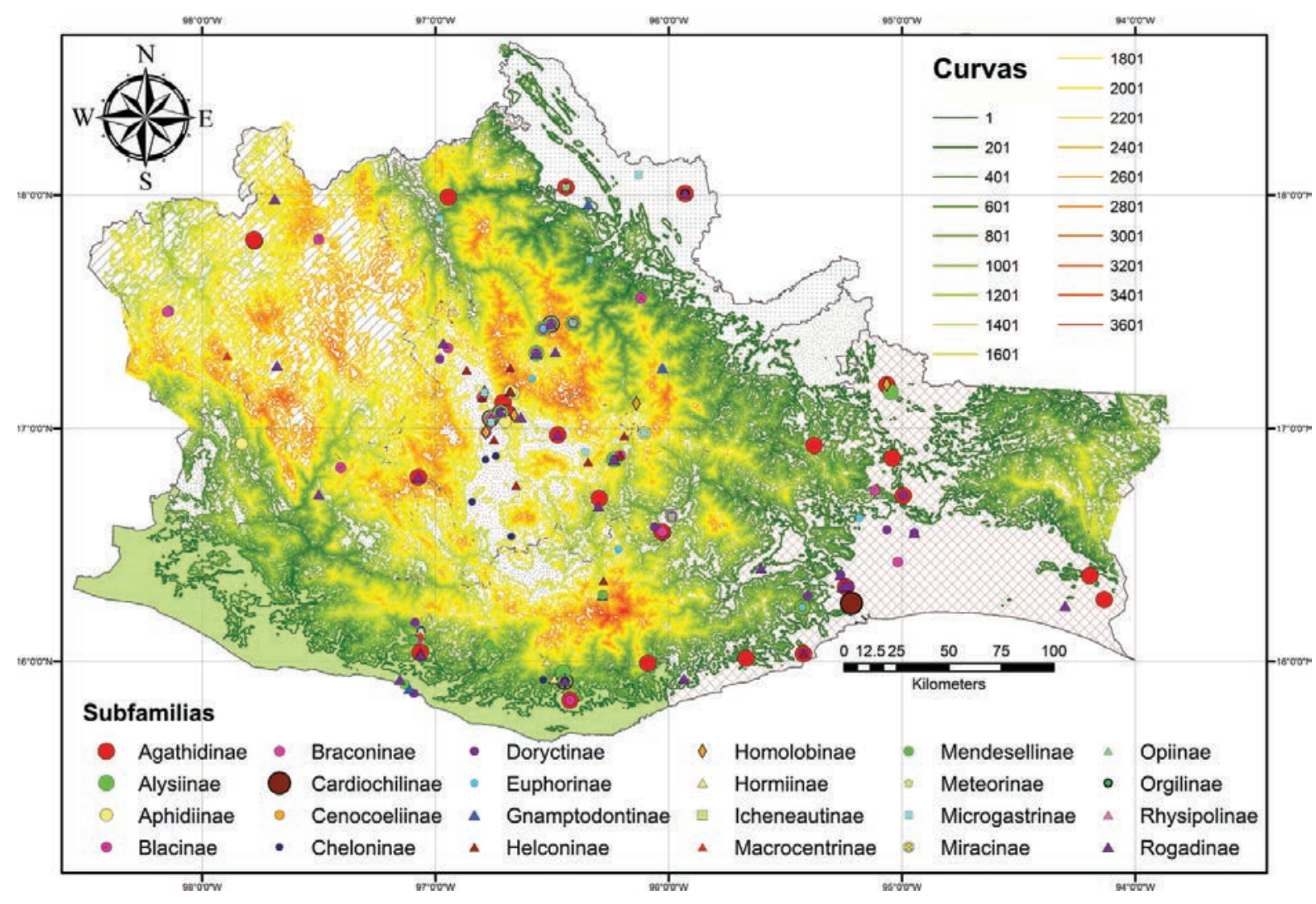

Figura 6. Registros de distribución de bracónidos sobre relieve del estado de Oaxaca.

La fauna mexicana ha recibido poca atención, por lo que estudios de revisión en los que se incorpore un análisis espacial de la distribución de las subfamilias de bracónidos nos ayudará a reconocer la biología de estos parasitoides según el comportamiento de estos insectos en diversos factores biogeográficos. Wharton et al. (1998) mencionan que la riqueza extrema de la fauna de los bracónidos del Nuevo Mundo, junto con el gran número de especies no descritas, ha limitado la habilidad de valorar la importancia de las relaciones biogeográficas para la mayoría de los taxa.

Pintado et al. (2000) mencionan que la cantidad de especies de bracónidos es más diversa y abundante en áreas tropicales, situación por la cual probablemente se obtuvieron más géneros para la región Costa, aunque; la abundancia descrita en este estudio no demuestra la riqueza de una región o del estado en general. Otro factor que influye en la distribución de bracónidos por regiones del estado se basa en que la composición de estos parasitoides cambia en zonas urbanas y perturbadas a diferencia de otras que no se encuentran bajo esa condición, debido a que esta familia en particular es muy susceptible a los cambios en su entorno (Delfín \& Burgos 2000), situación que habría que tomar en cuenta para trabajos posteriores. La abundancia de la subfamilia Doryctinae y Microgastrinae probablemente se deba a que ambas son de 
las más diversas de la familia Braconidae, existe un número inusual de géneros para los dorictinos en los trópicos, además que sus principales huéspedes son coleópteros (Marsh 1979). Asimismo, Microgastrinae tiene como principales huéspedes lepidópteros que son muy abundantes, esta subfamilia se encuentra en todos los continentes, desde clima tropical a clima ártico (Wharton et al. 1997).

Luna (2005) comenta que datos de vegetación y altitud son necesarios para conocer los requerimientos ecológicos de los organismos y el tipo de comunidades que éstas integran, es por estas razones que el presente estudio trata de dar a conocer la relación existente entre avispas parasitoides con atributos biogeográficos de Oaxaca, entre los que se incluye la vegetación del estado, esto por la importancia ecológica que tienen las especies de la familia Braconidae por ser agentes reguladores de diversos grupos de insectos fitófagos, sirviendo así también como indicadores de la presencia o ausencia de poblaciones de insectos perjudiciales (Lasalle 1993). White $\&$ Wilson (2012) comentan que el clima es una de las razones del fracaso al querer establecer insectos benéficos para el control de plagas, ya que las temperaturas sufren variaciones según la zona geográfica. Al respecto Hance et al. (2007) mencionan que en condiciones extremas ocurrirá un incremento en la abundancia de insectos plaga, debido posiblemente a una disminución en la actividad de los insectos parasitoides, perturbando la relación parasitoides - plaga y la capacidad de control. Otros estudios sugieren que altas temperaturas favorecerán a los insectos parasitoides en lugar de sus anfitriones (Davis et al. 1998, Kiritani 2006).

Harrington et al. (2001) \& Kiritani (2006) comentan que altas temperaturas en estaciones frías pueden estimular la reproducción de insectos, generando un crecimiento poblacional agresivo de hospederos para insectos benéficos, pero Kiritani (2006) menciona que el comportamiento de los huéspedes y parasitoides puede ser totalmente diferente ante el calentamiento global. Por su parte Kiritani (2006) comenta que habrá casos en los que las condiciones cálidas aumentarán la eficacia de enemigos naturales (Cammell \& Knight 1992, Kiritani 1999) o se dará el aumento de la vulnerabilidad de las presas (Awmack et al. 1997, Thomson et al. 2010). Durante los últimos 100 años el promedio global de temperatura ha incrementado aproximadamente en $0.6^{\circ} \mathrm{C}$, siendo el incremento más intenso durante el último siglo (Houghton et al. 2001).

Shou-Horng et al. (2010) comentan que el cambio climático puede realizar variaciones en el comportamiento de los insectos, mientras que Chu \& Chao (2000) mencionan que la temperatura es uno de los factores ambientales más importantes que tiene influencia directa sobre la distribución, desarrollo, sobrevivencia y reproducción de los insectos. Dependiendo de la estrategia de desarrollo que adopte un determinado espécimen la temperatura puede ejercer un efecto diferente en cada organismo (Bale et al. 2002). Kiritani (1988) sugiere que con bajas temperaturas, especies de climas tropicales y subtropicales que no guardan reposo en su ciclo de vida serán capaces de migrar a otros hábitats durante el tiempo que su resistencia al frío 
lo permita; por otra parte, especies de clima templado que se refugian en invierno, no podrán migrar hasta que las temperaturas se eleven los suficiente para poder hacerlo Chu \& Chao (2000). comentan que muchos insectos serán capaces de asimilar estos cambios, pero no sus plantas hospederas.

Parmesan \& Yohe (2003) realizaron una investigación en la que utilizaron 1700 especies de insectos, determinando que el $50 \%$ de estos organismos son afectados por el cambio climático y en un panorama futuro se predice que entre el 15 a $37 \%$ de esas especies pueden extinguirse en el año 2050 (Thomas et al. 2004). Los parasitoides e hiperparasitoides son organismos que se espera sean los más afectados, por la necesidad de desarrollarse de forma externa o interna en un hospedero (Godfray 1994).

Es indudable que el trabajo de investigación realizado en las cercanías de las carreteras es el que ha proporcionado el mayor conocimiento sobre la familia Braconidae, lo cual es comprensible pues las carreteras fungen como accesos viables a las localidades donde se encuentra la fauna y flora silvestre que sirven de hospederos y refugio a la diversidad de artrópodos (Lecler \& Rodríguez 1998).

AGRADECIMIENTOS. Al personal de las colecciones entomológicas revisadas. A la Secretaría de Investigación y Posgrado (SIP) del Instituto Politécnico Nacional por el financiamiento del proyecto SIP-20144658 Estudio de himenópteros parasitoides en Oaxaca y al Consejo Nacional de Ciencia y Tecnología (CONACYT) por el apoyo económico brindado para la realización del trabajo.

\section{LITERATURA CITADA}

Achterberg, C. 1979. A revision of the subfamily Zelinae auct. (Hymenoptera, Braconidae). Tijdschrift voor Entomologie, 122: 241-479.

Arce, G. F. \& García, J. G. 1991. Insectos benéficos que parasitan al gusano cogollero del maíz. Boletín No8. CIIDIR-Oaxaca, IPN. México. pp. 2.

Awmack, C. S., Woodcock, C. M. \& Harrington, R. 1997. Climate change may increase vulnerability of aphids to natural enemies. Ecological Entomology, 22: 366-368.

Bale, J. S., Masters, G. J., Hodkinson, I. D., Awmack, C., Bezemer, T. M., Brown, V. K., Butterfield, J., Buse, A., Coulson, J. C., Farrar, J., Good, J. E. G., Harrington, R., Hartley, S., Jones, T. H., Lindroth, R. L., Press, M. C., Symrnioudis, I., Watt, A. D. \& Whittaker, J. B. 2002. Herbivory in global climate change research: direct effects of rising temperatures on insect herbivores. Global Change Biology, 8: 1-16.

Barrera, J. F. 2008. Aplicación de la Ley del Poder de Taylor al Muestreo de Insectos. pp. 47-79. In: J. Toledo \& F. Infante (Eds.). Manejo Integrado de Plagas, Trillas, México.

Batalden, R. V., Oberhauser, K. \& Peterson, A. A. 2007. Ecological niches in sequential generations of eastern North American monarch butterflies (Lepidoptera: Danaidae): the ecology of migration and likely climate change implications. Environmental Entomology, 36: 1365-1373.

Briceño, G. R. A. 2003. Taxonomic revision of the genus Sesioctonus Viereck (Hymenoptera: Braconidae: Agathidinae). Journal of Hymenoptera Research, 12: 238-271.

Briggs, D. 2005. The role of GIS: coping with space (and time) in air pollution exposure assessment. Journal of Toxicology and Environmental Health, 68: 1243-1261. 
Cammell, M. E. \& Knight, J. D. 1992. Effects of climatic change on the population dynamics of crop pests. Advances in Ecological Research, 22: 117-162.

Cannon, R. J. C. 1998. The implications of predicted climate change for insect pests in the UK, with emphasis on non-indigenous species. Global Change Biology, 4: 785-796.

Cauich-Kumul, R., Delfín-Gonzalez, H., López-Martínez, V. \& Sharkey, M. 2012. Braconid Wasps (Hymenoptera: Braconidae) of Northern Yucatan, Mexico: Subfamilies Agathidinae and Doryctinae (excluding Heterospilus Haliday). Journal of the Kansas Entomological Society, 85: 186-205.

Chu, Y. I. \& Chao, J. T. 2000. The impact of global change on insects (in Chinese). pp. 341-366. In: T. C. Wang \& W. J. Wu (Eds.). Applied Entomology. National Taiwan University. Taipei, Taiwan.

Coronado-Blanco, J. M. 2013. La familia Braconidae (Hymenoptera) en México. pp. 31-46. In: A. Equihua M. et al. (Eds.). Entomología Mexicana Vol. 12, Tomo 1., Sociedad Mexicana de Entomología, México.

Coronado-Blanco, J. M. \& Zaldívar-Riverón, A. 2014. Biodiversidad de Braconidae (Hymenoptera: Ichneumonoidea) en México. Revista Mexicana de Biodiversidad, Sup. 85: S372-S378.

Cruz, S. E. 2009. Evaluación del parasitismo natural en Spodoptera frugiperda (Smith) (Lepidoptera: Noctuidae) en maíz. Tesis de Maestría en Ciencias. Instituto Politécnico Nacional, CIIDIR Oaxaca. $60 \mathrm{p}$.

Davis, A. J., Jenkinson, L. S., Lawton, J. H., Shorrocks, B. \& Wood, S. 1998. Making mistakes when predicting shifts in species range in response to global warming. Nature, 391: 783-786.

Deans, A. R., Whitfield, J. B. \& Janzen, D. H. 2003. Taxonomy and natural history of the microgastrinae genus Alphomelon Mason (Hymenoptera: Braconidae). Journal of Hymenoptera Research, 12: $1-41$.

Delfín, G. H. \& Wharton, R. A. 2000. Historical Review of the genera Aleiodes and Rogas in Mexico, with a redescription of Aleiodes cameronii (Hymenoptera: Braconidae). Pan-Pacific Entomologist, 76: 58-70.

Delfín, G. H. \& Burgos, R. 2000. Los bracónidos (Hymenoptera: Braconidae) como grupo parámetro en las selvas deciduas del trópico: Una discusión acerca de su posible uso. Acta Zoológica Mexicana (n.s.), 79: 43-56.

Delfín, G. H., Chay, D., González, A., Hernández, L. \& Suarez, C. 2002. New records of Braconidae (Hymenoptera) Subfamilies and genera from Mexico and the state of Yucatán. Transactions of the American Entomological Society, 128: 99-108.

Delfín-González, H. \& León-Burgos, F. A. 1997. Géneros de Braconidae (Hymenoptera) en Yucatán. Algunos elementos para el planteamiento de patrones de riqueza. Acta Zoológica Mexicana (n.s.), 70: 65-77.

Delfín-González, H., Chay-Hernández, D., González-Moreno, A., Hernández-Puch, L. \& SuárezCastillejos, C. 2002. New Records of Braconidae (Hymenoptera) Subfamilies and Genera from México and the State of Yucatan. Transactions of the American Entomological Society, 128: 99108.

Easterling, D. R., Meehl, G. A., Parmesan, C., Changnon, S. A., Karl, T. R. \& Mearns, L. O. 2000. Climate extremes: observations, modeling, and impacts. Science, 289: 2068-2074.

Ellsbury, M. M., Woodson, W. D., Clay, S. A., Malo, D., Schumacher, J., Clay, D. E. \& Carlson, C. G. 1998. Geostatistical characterization of the spatial distribution of adult corn rootworm emergence. Environmental Entomology, 27: 910-917.

ESRI. 2012. ArcGIS 10.1. ArcMap. Environmental Systems Research Institute Inc. USA.

Figueroa-De la Rosa, J. I., Sharkey, M. J., Romero N. ,J., Sánchez G., J. A., Martínez, A. M., López M., V. \& Pineda, S. 2011. Revision of the new world genus Crassomicrodus Ashmead (Hymenoptera, Braconidae, Agathidinae), with an identification key to species. ZooKeys, 142: 27-75. 
Fortier, J. C. 2006. Twelve new species and a new combination of the Aleiodes pilosus species-group (=Tetrasphaeropyx Ashmead) (Hymenoptera, Braconidae, Rogadinae) in North America: Part 1. Entomological News, 117: 465-484.

Fortier, J. C. 2007. Eight new species and a key to species of the Aleiodes pilosus species-group (=Tetrasphaeropyx Ashmead) (Hymenoptera, Braconidae, Rogadinae) in North America. Entomological News, 118: 11-30.

Fortier, J. C. 2009. A revision of the Tetrasphaeropyx Ashmead lineage of the genus Aleiodes Wesmael (Hymenoptera: Braconidae: Rogadinae. Monograph. Zootaxa, 2256: 1-126.

García-Mendoza, A. J., Ordóñez, M. J. \& Briones S., M. 2004. Biodiversidad de Oaxaca. Instituto de Biología, UNAM-Fondo Oaxaqueño para la conservación de la Naturaleza. World Wildlife Fund. México. pp.19-26.

Godfray, H. C. J. 1994. Parasitoids. Behavioral and Evolutionary Ecology. Princeton, N.J., Princeton Univ. Press. 473 pp.

González, H. A., Wharton, R. A., Sánchez, J. A., Delfín, H., Lomelí, J. R., López, V. \& Figueroa, J. I. 2003. Catálogo Ilustrado de Braconidae (Hymenoptera: Ichneumonoidea) de México. Universidad Autónoma de Nuevo León. CD-ROM.

Hance, T., van Baaren, J., Vernon, P. \& Boivin, G. 2007. Impact of extreme temperatures on parasitoids in a climate change perspective. Annual Review of Entomology, 52: 107-126.

Harrington, R., Fleming, R. \& Woiwod, I. 2001. Climate change impacts on insect management and conservation in temperate regions: can they be predicted? Agricultural and Forest Entomology, 3: 233-240.

Houghton, J. T., Ding, Y., Griggs, D. J., Noguer, M., van der Linden, P. J., Xiaosu, D., Maskell, K. \& Johnson, C. A. 2001. Climate Change 2001: The Scientific Basis. Cambridge University Press, Cambridge. 881 pp.

Janzen, D. H., Sharkey, M. J. \& Burns, J. M. 1998. Parasitization biology of a new species of Braconidae (Hymenoptera) feeding on larvae of Costa Rican dry forest skippers (Lepidoptera: Hesperiidae: Pyrginaae). Tropical Lepidoptera, 9: 33-41.

Jarquín-López, R., Martínez M., L., Sánchez G., J. A. \& Figueroa, J. I. 2011. Parasitoides asociados a Anthonomus sisyphus Clark (Coleóptera: Curculionidae) en frutos de Nanche Rojo (Malpighia mexicana) en Oaxaca, México. Southwestern Entomologist, 36: 351-364.

Jerez, V. 2000. Diversidad y patrones de distribución geográfica de insectos coleópteros en ecosistemas desérticos de la región de Antofagasta, Chile. Revista Chilena de Historia Natural, 73: 79-92.

Jerrett, M., Arain, A., Kanaroglou, P., Beckerman, B., Potoglou, D. \& Sahsuvaroglu, T. 2005. A review and evaluation of intraurban air pollution exposure models. Journal of Exposure Science and Environmental Epidemiology, 15: 185-204.

Kiritani, K. 1988. Effects of climate change on the insect fauna. Meteorol. Res. Rept., 162: 137-141.

Kiritani, K. 1999. Shift of IPM strategy for rice under global warming in temperate áreas. pp. 235-244. In: R. Zhang, D. Gu, W. Zhang, C. Zhou \& Y. Pang (Eds.). Integrated Pest Management in RiceBased Ecosystem. Zhongshan University Press, Guangzhou, PRC.

Kiritani, K. 2006. Predicting impacts of global warming on population dynamics and distribution of arthropods in Japan. Population Ecology, 48: 5-12.

Labougle, R. J. M. 1980. Análisis sobre la sistemática de la familia Braconidae (Insecta: Hymenoptera) y su situación actual en México. Tesis Facultad de Ciencias, Universidad Nacional Autónoma de México, México D. F. 185 p.

Lasalle, J. 1993. Parasitic Hymenoptera, biological control and diversity. Pp. 197-215. In: J. Lasalle \& I. D. Gauld (eds). Hymenoptera and Biodiversity. C. A. B. International, Wallingford. 
Lastuvka, Z. 2009. Climate change and its possible influence on the occurrence and importance of insect pests. Plant Protection Science, 45: S53-S62.

Lecler, G. \& Rodríguez, J. 1998. Using a GIS to determine critical areas in the central volcanic cordillera conservation área. pp. 108-126. In: B. G. Savitsky \& E. Lacher (Eds.). GIS Methodologies for Developing Conservstion Strategies: Tropical Forest Recovery and Wildlife Management in Costa Rica. Columbia University Press. New York.

López-Martínez, V., Figueroa De la R., J. I., Romero, J., Sánchez, J. A. \& Anaya, S. 2004. New host record for Urosigalphus mimosestes Gibson and first record of U. neomexicanus Crawford (Hymenoptera: Braconidae) in México. Entomological News, 115: 175-177.

López-Martínez, V., Romero N., J., Gonzalez H., A., Equihua M., A., Ramírez A., S. \& Sánchez G., J. A. 2003. Descripción del macho de Triaspis kurtogaster Martin y comentarios en la distribución de T. azteca Martin y T. eugenii Wharton y López-Martínez (Hymenoptera: Braconidae). Folia Entomológica Mexicana, 42: 153-160.

López-Martínez, V., Saavedra A., M., Delfín G., H., Figueroa De la Rosa, J. I. \& García R., M. J. 2009. New Neotropical Distribution Records of Braconid Wasps (Hymenoptera: Braconidae). Neotropical Entomology, 38: 213-218.

Luna, J. M. 2005. Técnicas de colecta y preservación de insectos. Boletín Sociedad Entomológica Aragonesa, 37: 385-408.

Marsh, P. M. 1979. Family Braconidae, pp. 144-295. In: Krombein, K. V., Hurd, P. D., Smith, D. R. \& Burks, B. D. (Eds.). Catalog of Hymenoptera in America North of Mexico. Smithsonian Inst. Press. Washington D.C.

Marsh, P. M. 1988. Revision of the Tribe Odontobraconini in the Western Hemisphere (Hymenoptera: Braconidae: Doryctinae). Systematic Entomology, 13: 443-464.

Marsh, P. M. 1993. Description of new Western Hemisphere genera of the subfamily Doryctinae (Hymenoptera: Braconidae). Contributions of the American Entomological Institute, 28: 1-58.

Marsh, P. M. \& Shaw, S. R. 2001. Revision of North American Aleiodes Wesmael (Part 6): The gasterator (Jurine) and Unipunctator (Thunberg) species-group (Hymenoptera: Braconidae: Rogadinae). Proceedings of the Entomological Society of Washington, 103: 291-307.

Martínez, G. A., Sánchez, J. A. \& Martínez, L. 2008. Bracónidos (Hymenoptera) presentes en Santa María Yavesía, Sierra Norte de Oaxaca. pp. 991-995. In: E. Estrada, A. Equihua, J. R. Padilla, \& A. Mendoza (eds). Entomología Mexicana V, Sociedad Mexicana de Entomología, México.

Martínez, J. J. \& Zaldívar-Riverón, A. 2013. Seven new species of Allorhogas (Hymenoptera: Braconidae: Doryctinae) from Mexico. Revista Mexicana de Biodiversidad, 84: 117-139.

Mercado, C. P. 1996. Parasitoides del descortezador suriano de los pinos Dendroctonus frontalis Zimm. (Coleóptera: Scolytidae) en Tlaxiaco, Oax., México. Tesis Universidad Autónoma Chapingo, Parasitología Agrícola, Chapingo, Estado de México. 66 pp.

Mercado, I. \& Wharton, R. A. 2003. Mexican cardiochiline genera (Hymenoptera: Braconidae), including a preliminary assessment of species-group in Toxoneuron Say and Retusigaster Dangerfield, Austin and Whitfield. Journal of Natural History, 37: 845-902.

Morales, L. M. \& Sánchez, J. A. 2007. Bracónidos (Hymenoptera) presentes en Pluma Hidalgo, Oaxaca. Pp. 1307-1311. In: Estrada, V. E. et al. (eds). Entomología Mexicana Vol. 6, Tomo 2, Sociedad Mexicana de Entomología, México.

Moral-García, F. J., Rodríguez, B. J., Arranz R., A., De la Cruz B., J. I. \& Honorio G., F. 2004. Técnicas geoestadísticas aplicadas al análisis de la distribución de capturas de Helicoverpa armígera (Hübner) (Lepidoptera: Noctuidae) mediante trampas con feromonas sexuales en una plantación de tomate. Boletín de Sanidad Vegetal de Plagas, 30: 733-744. 
Muesebeck, C. F. W. 1937. A new West Indian species of Mirax Haliday parasitic on the coffe leafminer (Hymenoptera: Braconidae). Proceedings of the Entomological Society of Washington, 39: 139-141.

Muesebeck, C. F. W. 1958. New Neotropical wasps of the family Braconidae (Hymenoptera) in the U. S. National Museum. Proceedings of the United States National Museum, 107: 405-461.

Ojeda, S. J. 2005. Estudio de la familia Braconidae asociada al cultivo de alfalfa (Medicago sativa L.) en Santa Cruz Xoxocotlán, Oaxaca. Tesis Profesional. Instituto Tecnológico Agropecuario de Oaxaca No. 23. 51 p.

Park, Y. \& Obrycki, J. J. 2004. Spatio-temporal distribution of corn leaf Aphids (Homoptera: Aphididae) and lady beetles (Coleoptera: Coccinellidae) in Iowa cornfields. Biological Control, 31: 210217.

Parmesan, C. \& Yohe, G. 2003. A globally coherent fingerprint of climate change impacts across natural systems. Nature, 421: 37-42.

Parmesan, C., Ryrholm, N., Stefanescu, C., Hill, J. K., Thomas, C. D., Descimon, H., Huntley, B., Kaila, L., Kullberg, J., Tammaru, T., Tennent, W. J., Thomas, J. A. \& Warren, M. 1999. Poleward shifts in geographical ranges of butterfly species associated with regional warming. Nature, 399: 579-583.

Pintado, C. B., Carrasco, V. D. \& López, M. C. 2000. Distribución de Braconidae en áreas tropicales. Memorias del XXVIII Congreso Nacional de Entomología. 83 pp.

Quicke, D. L. J. \& Kruft, R. A. 1995. Species of Yelicones (Hymenoptera: Braconidae: Rogadinae) in North America with descriptions of two new species. Annals of the Entomological Society of America, 88: 129-138.

Quicke, D. L. J., Chishti, M. J. K. \& Basibuyuk, H. H. 1996. A revision of the Yelicones species (Hymenoptera: Braconidae: Rogadinae) from Central America, with descriptions of sixteen new species. Zool. Med. Leiden, 70: 17-61.

Rodríguez-Leyva, E. 2007. Life History of Triaspis eugenii Wharton and López-Martínez (Hymenoptera: Braconidae) and Evaluation of its Potential for Biological Control of Pepper Weevil Anthonomus eugenii Cano (Coleoptera: Curculionidae). Ph.D. Dissertation, University of Florida, Gainesville. 110 pp.

Sánchez, G. J. A., Romero, J., Ramírez, S., Anaya, S. \& Carrillo, J. L. 1998. Géneros de Braconidae del estado de Guanajuato (Insecta: Hymenoptera). Acta Zoológica Mexicana (n.s.), 74: 59-137.

Sánchez, G. J. A. \& López, V. 2000. Géneros de Braconidae (Insecta: Hymenoptera) depositados en la Colección Entomológica del Instituto de Fitosanidad del Colegio de Postgraduados. Acta Zoológica Mexicana (n.s.), 74: 59-137.

Sánchez, G. J. A., López, V., Figueroa, J. I., González, A. \& Pérez, R. 2003a. Revisión de Braconidae (Hymenoptera) del estado de Oaxaca, México. Pp. 789-792. In: J. Romero, E. G. Estrada \& A. Equihua (Eds.). Entomología Mexicana Vol. 2. Sociedad Mexicana de Entomología, México.

Sánchez, G. J. A., Wharton, R. A., Romero N., J., Gonzáles H., A., López M., V., Equihua M., A., Gonzáles H., H. \& Carrillo S., J. L. 2003b. Description of a new species of Blacus Nees (Hymenoptera: Braconidae) from México, utilizing characters of the male external genitalia. Pan-Pacific Entomologist, 79: 135-144.

Sánchez, G. J. A., Avendaño, S., Ojeda, J., Martínez, L., Coello, M. \& Pérez, R. 2005. Bracónidos (Hymenoptera) asociados a plagas en el estado de Oaxaca, México. Pp. 911-914. In: M. Morales, A. Mendoza, M. P. Ibarra \& S. Stanford (Eds.). Entomología Mexicana. Vol. 4, Sociedad Mexicana de Entomología, México. 
Sánchez, G. J. A., Morales, M. \& Martínez, A. 2008. Géneros de Braconidae (Hymenoptera) del estado de Oaxaca. Pp. 985-990. In: Estrada, V. E. G. et al. (eds). Entomología Mexicana. Vol. 7, Sociedad Mexicana de Entomología, México.

Sánchez, G. J. A., Morales, M., Martínez, A. \& Figueroa, J. I. 2009. Catálogo de bracónidos (Hymenoptera) del estado de Oaxaca, México. Pp. 907-912. In: E. G. Estrada et al. (Eds.). Entomología Mexicana. Vol. 8. Sociedad Mexicana de Entomología, México.

Sánchez, G. J. A., Martínez M., L., Figueroa-De la R., J. I. \& Jarquín L., R. 2010a. Control Biológico de Plagas en el Estado de Oaxaca. Pp. 142-145. In: V. M. Coria A., M. Lara Ch., G. Orozco G., H. J. Muñoz-Flores \& R. Sánchez M. (Eds.). Memoria de XXXIII Congreso Nacional de Control Biológico. Campo Experimental Uruapan, Uruapan, Michoacán, México.

Sánchez, G. J. A., Wharton, R. A., Romero N., J., Figueroa De la Rosa, J. I., López M., V., Martínez, A. M., Pérez P., R. \& Pineda, S. 2010b. Four new species of Blacus Nees (Hymenoptera, Braconidae, Blacinae) from Mexico. Zootaxa, 2641: 37-46.

Santiaguillo-Hernández, J. F., Cedillo-Portugal, E. \& Cuevas-Sánchez, J. A. 2010. Distribución Geográfica de Physalis spp. en México. Publicaciones de la Red de tomate de cascara. Prometeo editores S.A. de C.V. Universidad Autónoma Chapingo, México-Texcoco. 245 pp.

Sharkey, M. J. 1988. A taxonomic revision of Alabagrus (Hymenoptera: Braconidae). Bulletin of the British Museum (Natural History), 57: 311-437.

Shou-Horng, H., Ching H., Ch. \& Wen J., W. 2010. Possible Impacts of Climate Change on Rice Insect Pests and Management Tactics in Taiwan. Crop. Environment and Bioinformatics, 7: 269-279.

Starý, P. 1983. New species and records of aphid parasitoids from Mexico (Hymenoptera, Aphidiidae). Acta Entomologica Bohemoslovaca, 80: 35-48.

Thomas, C. D., Cameron, A., Green, R. E., Bakkenes, M. \& Beaumont, L. J. 2004. Extinction risk from climate change. Nature, 427: 145-148.

Thomson, L. J., Macfadyen, S. \& Hoffmann, A. A. 2010. Predicting the effects of climate change on natural enemies of agricultural pests. Biological Control, 52: 296-306.

Vílchez, J. G. 2000. Introducción a los sistemas de información geoespacial. Consejo de Desarrollo Científico y Humanístico, Universidad de los Andes, Talleres Gráficos Universitarios, Mérida. 203 p.

Wharton, R. A., Marsh, P. M. \& Sharkey, M. J. 1997. Manual of the New World genera of the Family Braconidae (Hymenoptera). The International Society of Hymenopterists, Washington D.C. 447 p.

Wharton, R. A., Marsh, P. M. \& Sharkey, M. J. 1998. Manual para los géneros de la familia Braconidae (Hymenoptera) del nuevo mundo. The International Society of Hymenopterists. Washington, DC. pp. 1-447.

Wharton, R. A. \& Mercado, I. 2000. Braconidae (Hymenoptera). Pp. 635-647. In: B. J. llorente, S. E. González \& N. Papavero (eds). Biodiversidad, taxonomía y biogeografía de artrópodos de México. Hacia una síntesis de su conocimiento. Vol. II. UNAM-CONABIO-BAYER, México.

White, W. H. \& Wilson, L. T. 2012. Feasibility of using an alternative larval host and host plants to establish Cotesia flavipes (Hymenoptera: Braconidae) in the Temperate Louisiana Sugarcane Ecosystem. Environmental Entomology, 41: 275-281.

Whitfield, J. B. \& Mason, W. R. M. 1994. Mendesellinae, a new subfamily of braconid wasps (Hymenoptera, Braconidae) with a review of relationships within the microgastroid assemblage. Systematic Entomology, 19: 61-76.

Whitfield, J. B. \& Oltra, M. T. M. 2004. The Neotropical species of Deuterixys Mason (Hymenoptera: Braconidae). Journal of Hymenoptera Research, 13: 134-148.

Whitfield, J. B. 1990. Phylogenetic review of the Stiropius group of genera (Hymenoptera: Braconidae: Rogadinae) with description of a new Neotropical genus. Proc. Entomol. Soc. Wash., 92: 36-43. 
Williams, D. J. 2004. Revision of the genus Pseudognaptodon Fischer (Hymenoptera: Braconidae: Gnamptodontinae). Journal of Hymenoptera Research, 13: 149-205.

Woiwod, I. P. 1997. Detecting the effects of climate change on Lepidoptera. J. Insect Conserv., 1: 149158.

Yu, D. S., Achterberg C., C. \& Horstmann, K. 2012. World Ichneumonoidea 2011. Taxonomy, Biology, Morphology and Distribution (Braconidae). Ottawa: Taxapad (Scientific Names for Information Management) Interactive Catalogue. DVD/CD-ROM.

Zhou, X., Harrington, R., Woiwod, I. P., Perry, J. N., Bale, J. S. \& Clark, S. J. 1995. Effects of temperature on aphid phenology. Global Change Biology, 1: 303-313. 